\title{
RATIONALITY OF DESCENDENT SERIES FOR HILBERT AND QUOT SCHEMES OF SURFACES
}

\author{
DREW JOHNSON, DRAGOS OPREA, AND RAHUL PANDHARIPANDE
}

\begin{abstract}
Quot schemes of quotients of a trivial bundle of arbitrary rank on a nonsingular projective surface $X$ carry perfect obstruction theories and virtual fundamental classes whenever the quotient sheaf has at most 1-dimensional support. The associated generating series of virtual Euler characteristics was conjectured to be a rational function in OP1 when $X$ is simply connected. We conjecture here the rationality of more general descendent series with insertions obtained from the Chern characters of the tautological sheaf. We prove the rationality of descendent series in Hilbert scheme cases for all curve classes and in Quot scheme cases when the curve class is 0.
\end{abstract}

\section{Contents}

0. Introduction

1. Virtual Euler characteristics: Theorem 1

2. Descendent series of punctual Quot schemes: Theorem 2

3. Descendent series for the Hilbert scheme: Theorem 3

References

\section{INTRODUCTION}

0.1. Motivation. Let $X$ be a nonsingular projective surface, and let $X^{[n]}$ denote the Hilbert scheme of points. A well-known formula of Göttsche Gö] expresses the topological Euler characteristics of the Hilbert schemes in terms of the Dedekind eta function

$$
\sum_{n=0}^{\infty} \mathrm{e}\left(X^{[n]}\right) q^{n}=\left(q^{-\frac{1}{24}} \eta(q)\right)^{-\mathrm{e}(X)} .
$$

Göttsche's formula reflects the action of the Heisenberg algebra on the cohomology of $X^{[n]}$ constructed by $\left.\mathrm{Gr}, \mathrm{N}\right]$.

There are at least two possible directions of extending (11). First, we may view $X^{[n]}$ as the moduli space of rank 1 sheaves with trivial determinant. The higher rank moduli spaces of sheaves over $X$ play a central role in Vafa-Witten theory [TT, WW]. Explicit expressions for the generating series of the rank 2 and 3 moduli spaces are conjectured in

Date: March 2021. 
GK1, GK2]. Since the higher rank moduli spaces may be singular, the Euler characteristics are replaced by virtual analogues which take into account the deformation theory of the moduli space.

In a different direction, we may promote $X^{[n]}$ to more general Hilbert and Quot schemes and study the corresponding virtual invariants.

0.2. Quot schemes and virtual Euler characteristics. Let $X$ be a nonsingular projective surface, let $\beta \in H_{2}(X, \mathbb{Z})$ be an effective curve class of $X$, and let $N \geq 1$ be an integer. Consider the Quot scheme $\operatorname{Quot}_{X}\left(\mathbb{C}^{N}, \beta, n\right)$ parameterizing short exact sequences

$$
0 \rightarrow S \rightarrow \mathbb{C}^{N} \otimes \mathcal{O}_{X} \rightarrow Q \rightarrow 0
$$

where

$$
\operatorname{rank} Q=0, c_{1}(Q)=\beta, \chi(Q)=n .
$$

As explained in [MOP1, OP1], Quot ${ }_{X}\left(\mathbb{C}^{N}, \beta, n\right)$ carries a canonical 2-term perfect obstruction theory and a virtual fundamental class of dimension

$$
\operatorname{vdim}=\chi(S, Q)=N n+\beta^{2} .
$$

The virtual fundamental class of the Quot scheme was used in [MOP1] to prove Lehn's conjecture [Le for $K 3$ surfaces 1$]$

The virtual Euler characteristic is defined using the virtual tangent complex of the canonical obstruction theory [FG]. By analogy with the Poincaré-Hopf theorem, we set

$$
\mathrm{e}^{\mathrm{vir}}\left(\operatorname{Quot}_{X}\left(\mathbb{C}^{N}, \beta, n\right)\right)=\int_{\left[\operatorname{Quot}_{X}\left(\mathbb{C}^{N}, \beta, n\right)\right]^{\mathrm{vir}}} c\left(T^{\mathrm{vir}} \text { Quot }\right) \in \mathbb{Z},
$$

where $c$ denotes the total Chern class. The virtual tangent bundle is given by

$$
T^{\mathrm{vir}} \text { Quot }=\operatorname{Ext}_{X}^{\bullet}(S, Q)
$$

at each short exact sequence (2).

The generating series of virtual Euler characteristics,

$$
Z_{X, N, \beta}=\sum_{n \in \mathbb{Z}} \mathrm{e}^{\operatorname{vir}}\left(\operatorname{Quot}_{X}\left(\mathbb{C}^{N}, \beta, n\right)\right) q^{n},
$$

was introduced and studied in $\mathrm{OP} 1$. For fixed $X, N$, and $\beta$,

$$
\operatorname{vdim} \operatorname{Quot}_{X}\left(\mathbb{C}^{N}, \beta, n\right)=N n+\beta^{2}<0
$$

for $n$ sufficiently negative, hence $Z_{X, N, \beta}$ has a finite polar part. The following rationality property was conjectured 2 in OP1].

\footnotetext{
${ }^{1}$ See MOP2, MOP3, V for further developments.

${ }^{2}$ The conjecture can also be made for surfaces which are not simply connected, but we will not study non simply connected surfaces here (except in the $\beta=0$ case).
} 
Conjecture 1. Let $X$ be a nonsingular projective simply connected surface, and let $\beta$ be an effective curve class. The series $Z_{X, N, \beta}$ is the Laurent expansion of a rational function in $q$.

Conjecture 1 is known to be true in the following five cases:

- For all $N \geq 1$, the series $Z_{X, N, \beta}$ is rational if

(i) $X$ is any surface and $\beta=0$ OP1],

(ii) $X$ is a surface of general type 3 with $p_{g}>0$ and $\beta$ is any effective curve class [L, OP1],

(iii) $X$ is an elliptic surfacet with $p_{g}>0$ [L, OP2].

- For $N=1$, the series $Z_{X, 1, \beta}$ is also rational if

(iv) $X$ is a blow-up and $\beta$ is a multiple of the exceptional divisor [L, OP1],

(v) $X$ is a $K 3$ surface with reduced invariants for primitive curve classes [OP1].

Our first result here is a resolution of Conjecture 1 in case $N=1$.

Theorem 1. Let $X$ be a nonsingular projective simply connected surface, and let $\beta$ be an effective curve class. The generating series $Z_{X, 1, \beta}$ of virtual Euler characteristics is the Laurent expansion of a rational function in $q$.

In the $N=1$ case, the Quot scheme Quot ${ }_{X}\left(\mathbb{C}^{1}, \beta, n\right)$ is simply a Hilbert scheme of points and curves in $X$. Theorem 1 is therefore about the virtual Euler characteristics of such Hilbert schemes of surfaces. A crucial idea in our proof is to transform the geometry to the moduli space of stable pairs [PT1, PT2] on surfaces and to use the associated Jacobian fibration.

0.3. Rationality of descendent series. How special is the rationality of the generating series (3) of virtual Euler characteristics? We propose here a wider rationality statement for descendent series.

Let $X$ be a nonsingular projective simply connected surface, and let Quot ${ }_{X}\left(\mathbb{C}^{N}, \beta, n\right)$ be the Quot scheme parameterizing quotients (2). Let

$$
\begin{aligned}
& \pi_{1}: \operatorname{Quot}_{X}\left(\mathbb{C}^{N}, \beta, n\right) \times X \rightarrow \operatorname{Quot}_{X}\left(\mathbb{C}^{N}, \beta, n\right), \\
& \pi_{2}: \operatorname{Quot}_{X}\left(\mathbb{C}^{N}, \beta, n\right) \times X \rightarrow X
\end{aligned}
$$

be the two projections. Let

$$
\mathcal{Q} \rightarrow \operatorname{Quot}_{X}\left(\mathbb{C}^{N}, \beta, n\right) \times X
$$

\footnotetext{
${ }^{3}$ Property (ii) is proven in OP1 for simply connected minimal surfaces of general type with $p_{g}>0$ and a nonsingular canonical divisor. The assumptions other than $p_{g}>0$ were removed in $[\mathrm{L}$. A similar analysis was done in $\left[\mathrm{L}\right.$ at the level of $\chi_{-y}$-genera.

${ }^{4}$ Property (iii) is proven in OP2 for simply connected minimal elliptic surfaces. These assumptions were removed in $\left[\mathrm{L}\right.$ at the level of $\chi_{-y}$-genera.
} 
be the universal quotient. For a $K$-theory class $\alpha \in K^{0}(X)$, we define

$$
\alpha^{[n]}=\mathbf{R} \pi_{1 *}\left(\mathcal{Q} \otimes \pi_{2}^{*} \alpha\right) \in K^{0}\left(\operatorname{Quot}_{X}\left(\mathbb{C}^{N}, \beta, n\right)\right) .
$$

A generalization of the series (3) of virtual Euler characteristics is defined as follows. Let $\alpha_{1}, \ldots, \alpha_{\ell} \in K^{0}(X)$, and let $k_{1}, \ldots, k_{\ell}$ be non-negative integers. Set

$$
\begin{aligned}
& Z_{X, N, \beta}\left(\alpha_{1}, \ldots, \alpha_{\ell} \mid k_{1}, \ldots, k_{\ell}\right)= \\
& \sum_{n \in \mathbb{Z}} q^{n} \cdot \int_{\left[\operatorname{Quot}_{X}\left(\mathbb{C}^{N}, \beta, n\right)\right]^{\mathrm{vir}}} \operatorname{ch}_{k_{1}}\left(\alpha_{1}^{[n]}\right) \cdots \operatorname{ch}_{k_{\ell}}\left(\alpha_{\ell}^{[n]}\right) c\left(T^{\mathrm{vir}} \text { Quot }\right) .
\end{aligned}
$$

The Chern characters in (4) may be viewed as descendent insertions. Hence, we view $Z_{X, N, \beta}\left(\alpha_{1}, \ldots, \alpha_{\ell} \mid k_{1}, \ldots, k_{\ell}\right)$ as a descendent series.

Conjecture 2. The descendent series $Z_{X, N, \beta}\left(\alpha_{1}, \ldots, \alpha_{\ell} \mid k_{1}, \ldots, k_{\ell}\right)$ is the Laurent expansion of a rational function in $q$.

We can prove Conjecture 2 in case either $\beta=0$ or $N=1$.

Theorem 2. Let $X$ be a nonsingular projective surface. For $\beta=0$, the series

$$
Z_{X, N, 0}\left(\alpha_{1}, \ldots, \alpha_{\ell} \mid k_{1}, \ldots, k_{\ell}\right) \in \mathbb{Q}((q))
$$

is the Laurent expansion of a rational function in $q$.

Theorem 3. Let $X$ be a nonsingular projective simply connected surface, and let $\beta$ be an effective curve class. For $N=1$, the series

$$
Z_{X, 1, \beta}\left(\alpha_{1}, \ldots, \alpha_{\ell} \mid k_{1}, \ldots, k_{\ell}\right) \in \mathbb{Q}((q))
$$

is the Laurent expansion of a rational function in $q$.

The rationality statements for surfaces here are parallel to the rationality of the descendent series for stable pairs on 3-folds, see [P] for a survey and [PP1, [PP2, PP3, [PT1, PT2] for foundational results. Whether the descendent series (4) satisfy relations such as the Virasoro constraints for stable pairs $\mathrm{OOP}, \mathrm{MOOP}$ is an interesting question for further study.

Descendent integrals against the (non-virtual) fundamental class of the Hilbert scheme of points of a surface have been studied by Carlsson [C]; the descendent series are proven to be quasi-modular. The virtual fundamental class regularizes the descendent geometry in two ways: the theory can be defined more generally for Quot schemes of quotients supported on curves and the answers are rational functions.

The study of the virtual invariants of Quot schemes of surfaces can also be considered in $K$-theory. For recent results and conjectures related to the rationality of descendent series in $K$-theory, see AJLOP. Section 3.2 of AJLOP also contains subsequent developments regarding Conjecture 2 for all surfaces with $p_{g}>0$. 
0.4. Acknowledgments. Our study of the virtual Euler characteristics of the Quot scheme of surfaces was motivated in part by the Euler characteristic calculations of L. Göttsche and M. Kool [GK1, GK2] for the moduli spaces of rank 2 and 3 stable sheaves on surfaces. We thank A. Marian, W. Lim, A. Oblomkov, A. Okounkov, and R. Thomas for related discussions.

D. J. was supported by SNF-200020-182181. D. O. was supported by the NSF through grant DMS 1802228. R.P. was supported by the Swiss National Science Foundation and the European Research Council through grants SNF-200020-182181, ERC-2017-AdG786580-MACI, SwissMAP, and the Einstein Stiftung. We thank the Shanghai Center for Mathematical Science at Fudan University for a very productive visit in September 2018 at the start of the project.

The project has received funding from the European Research Council (ERC) under the European Union Horizon 2020 Research and Innovation Program (grant No. 786580).

\section{Virtual Euler characteristics: Theorem 1}

1.1. Obstruction theory. We start the proof of Theorem 1 with an explicit description of the Hilbert scheme and the obstruction theory in the $N=1$ case.

Let $X$ be a nonsingular projective surface. When $N=1$, the following isomorphism was proved in $[\mathrm{F}]$ :

$$
\operatorname{Quot}_{X}\left(\mathbb{C}^{1}, \beta, n\right) \simeq X^{[m]} \times \operatorname{Hilb}_{\beta} .
$$

Here, $X^{[m]}$ is the Hilbert scheme of $m$ points of $X, \mathrm{Hilb}_{\beta}$ is the Hilbert scheme of divisors of $X$ in the class $\beta$, and

$$
m=n+\frac{\beta\left(\beta+K_{X}\right)}{2} .
$$

Under this isomorphism, each pair $(Z, D) \in X^{[m]} \times \mathrm{Hilb}_{\beta}$ yields a short exact sequence

$$
0 \rightarrow I_{Z}(-D) \rightarrow \mathcal{O}_{X} \rightarrow Q \rightarrow 0
$$

The Hilbert scheme Hilb $\beta$ parameterizes only pure dimension 1 subschemes. There is an Abel-Jacobi map

$$
\operatorname{AJ}_{\beta}: \operatorname{Hilb}_{\beta} \rightarrow \operatorname{Pic}^{\beta}(X), \quad D \mapsto \mathcal{O}_{X}(D),
$$

with fibers given by projective spaces of possibly varying dimension. As noted in [DKO], $\mathrm{Hilb}_{\beta}$ carries a virtual fundamental class of dimension

$$
\operatorname{vim}_{\beta}=\frac{\beta\left(\beta-K_{X}\right)}{2} .
$$

The virtual fundamental class of $\operatorname{Quot}_{X}\left(\mathbb{C}^{1}, \beta, n\right)$ was identified in $[\mathrm{L}$ to equal

$$
\left[\operatorname{Quot}_{X}\left(\mathbb{C}^{1}, \beta, n\right)\right]^{\mathrm{vir}}=\mathrm{e}(\mathrm{B}) \cap\left(\left[X^{[m]}\right] \times\left[\mathrm{Hilb}_{\beta}\right]^{\mathrm{vir}}\right)
$$


where

$$
\mathrm{B}=\mathrm{RHom}_{\pi}\left(\mathcal{O}_{\mathcal{W}}, \mathcal{O}(\mathcal{D})\right)
$$

Here

$$
\mathcal{W} \subset X \times X^{[m]}, \mathcal{D} \subset X \times \operatorname{Hilb}_{\beta}
$$

are the universal families, and

$$
\pi: X \times X^{[m]} \times \operatorname{Hilb}_{\beta} \rightarrow X^{[m]} \times \mathrm{Hilb}_{\beta}
$$

is the projection.

When $X$ is simply connected, the Hilbert scheme $\mathrm{Hilb}_{\beta}=\mathbb{P}$ is a projective space of dimension $h^{0}(\beta)-1$. The obstruction bundle for $\operatorname{Quot}_{X}\left(\mathbb{C}^{1}, \beta, n\right)$ given above simplifies to the expression found in [OP1]:

$$
\text { Obs }=\left(H^{1}(M)-H^{0}(M)\right) \otimes \mathcal{L}+\left(M^{[m]}\right)^{\vee} \otimes \mathcal{L}+\mathbb{C}^{p_{g}} .
$$

Here

$$
M=K_{X}-\beta
$$

and the superscript ( $)^{[m]}$ denotes the usual tautological bundle over the Hilbert scheme of points $X^{[m]}$. Furthermore,

$$
\mathcal{L}=\mathcal{O}_{\mathbb{P}}(1) .
$$

Theorem 1 is established whenever $p_{g}>0$. For surfaces of positive Kodaira dimension, the claim follows by cases (ii) and (iii) discussed after Conjecture 1 in Section 0.2. The only remaining cases are $K 3$ surfaces and their successive blowups. Invariants of $K 3$ surfaces vanish unless $\beta=n=0$, see [MOP1]. Theorem 6 of [L] determines the invariants of blowups in terms of explicit rational functions, see also Section 3.2 .5 below.

We assume $p_{g}=0$ for the remainder of Section 1 . Since $\beta$ is an effective curve class, the condition $p_{g}=0$ implies

$$
H^{0}(M)=H^{0}\left(K_{X}-\beta\right)=0 .
$$

The obstruction bundle therefore further simplifies to

$$
\text { Obs }=H^{1}(M) \otimes \mathcal{L}+\left(M^{[m]}\right)^{\vee} \otimes \mathcal{L} .
$$

1.2. Rationality. For a nonsingular scheme $S$ endowed with a perfect obstruction theory and obstruction bundle Obs, the virtual Euler characteristic is given by

$$
\mathrm{e}^{\mathrm{vir}}(S)=\int_{M} \mathrm{e}(\mathrm{Obs}) \cdot \frac{c(T S)}{c(\text { Obs })} .
$$

In our situation (assuming $p_{g}=0$ ),

$$
\mathrm{e}^{\mathrm{vir}}\left(\operatorname{Quot}_{X}\left(\mathbb{C}^{1}, \beta, n\right)\right)=\int_{X^{[m]} \times \mathbb{P}} c_{1}(\mathcal{L})^{h^{1}(\beta)} \cdot \mathrm{e}\left(\mathcal{L} \otimes\left(M^{[m]}\right)^{\vee}\right) \cdot \frac{c\left(T X^{[m]}\right) \cdot c(\mathcal{L})^{\chi(\beta)}}{c\left(\mathcal{L} \otimes\left(M^{[m]}\right)^{\vee}\right)}
$$


We can integrate out the hyperplane class to reduce the dimension of the projective space to $\chi(\beta)-1$. Theorem 1 follows from the following result.

Proposition 1. Let $V$ be a finite dimensional vector space, and let $M \rightarrow X$ be a line bundle over a nonsingular projective surface. The series

$$
Z_{X, M, V}=\sum_{n=0}^{\infty} q^{n} \cdot \int_{X^{[n]} \times \mathbb{P}(V)} \mathrm{e}\left(\mathcal{L} \otimes\left(M^{[n]}\right)^{\vee}\right) \cdot \frac{c\left(T X^{[n]}\right) \cdot c(T \mathbb{P}(V))}{c\left(\mathcal{L} \otimes\left(M^{[n]}\right)^{\vee}\right)}
$$

is a rational function in $q$.

In fact, we will prove a stronger claim. For a rank $r$ vector bundle $E \rightarrow S$ over a scheme $S$ with Chern roots $x_{1}, \ldots, x_{r}$, define

$$
P_{d}(E)=\sum_{i=1}^{r} \frac{1}{\left(1+x_{i}\right)^{d}}
$$

For a finite sequence $B=\left(b_{1}, \ldots, b_{\ell}\right)$ of non-negative integers, we set

$$
P(E, B)=\prod_{i=1}^{\ell} P_{i}(E)^{b_{i}}
$$

Write

$$
Z_{X, M}[a, B]=\sum_{n=0}^{\infty} q^{n} \cdot \int_{X^{[n]}} c_{n-a}\left(\left(M^{[n]}\right)^{\vee}\right) \cdot c\left(T X^{[n]}\right) \cdot \frac{P\left(\left(M^{[n]}\right)^{\vee}, B\right)}{c\left(\left(M^{[n]}\right)^{\vee}\right)}
$$

Proposition 2. For all pairs $(X, M)$, non-negative integers a, and finite sequences $B$, the series $Z_{X, M}[a, B]$ is a rational function in $q$.

Proposition 2 implies Proposition 1 by the following argument. Let $\zeta=c_{1}(\mathcal{L})$ denote the hyperplane class on $\mathbb{P}(V)$. We analyze the expressions appearing in Proposition 1 . First,

$$
\mathrm{e}\left(\mathcal{L} \otimes\left(M^{[n]}\right)^{\vee}\right)=\sum_{a=0}^{n} \zeta^{a} \cdot c_{n-a}\left(\left(M^{[n]}\right)^{\vee}\right)
$$

Next, we write $x_{1}, \ldots x_{n}$ for the Chern roots of $M^{[n]}$. We have

$$
\frac{1}{c\left(\mathcal{L} \otimes\left(M^{[n]}\right)^{\vee}\right)}=\prod_{i=1}^{n} \frac{1}{1-x_{i}+\zeta}
$$

We expand

$$
\frac{1}{1-x_{i}+\zeta}=\frac{1}{1-x_{i}} \cdot \sum_{j=0}^{\infty}(-1)^{j} \cdot \zeta^{j}\left(1-x_{i}\right)^{-j}
$$


which yields

where

$$
\frac{1}{c\left(\mathcal{L} \otimes\left(M^{[n]}\right)^{\vee}\right)}=\frac{1}{c\left(\left(M^{[n]}\right)^{\vee}\right)} \cdot\left(\sum_{j=0}^{\infty}(-1)^{j} \zeta^{j} H_{j}\right)
$$

$$
H_{j}=\sum_{j_{1}+\ldots+j_{n}=j}\left(1-x_{1}\right)^{-j_{1}} \cdots\left(1-x_{n}\right)^{-j_{n}}
$$

The integral in Proposition 1 becomes

$$
\int_{X^{[n]} \times \mathbb{P}(V)}\left(\sum_{a=0}^{n} \zeta^{a} \cdot c_{n-a}\left(\left(M^{[n]}\right)^{\vee}\right)\right) \cdot \frac{c\left(T X^{[n]}\right)}{c\left(\left(M^{[n]}\right)^{\vee}\right)} \cdot\left(\sum_{j=0}^{\infty}(-1)^{j} \zeta^{j} H_{j}\right) \cdot(1+\zeta)^{v}
$$

where $\operatorname{dim} V=v$.

After integrating out $\zeta$ over $\mathbb{P}(V)$, we are led to expressions of the form

$$
\int_{X^{[n]}} c_{n-a}\left(\left(M^{[n]}\right)^{\vee}\right) \cdot \frac{c\left(T X^{[n]}\right)}{c\left(\left(M^{[n]}\right)^{\vee}\right)} \cdot H_{j}
$$

with $a+j \leq v-1$. Crucially, both $a$ and $j$ are bounded by $\operatorname{dim} V=v$, independently of $n$. Furthermore, each $H_{j}$ is symmetric in the Chern roots so can be expressed as a polynomial in the power sums

$$
P_{d}=\sum_{i=1}^{n} \frac{1}{\left(1-x_{i}\right)^{d}}
$$

in a fashion which is independent of $n$. Explicitly, we have

$$
\sum_{j=0}^{\infty} t^{j} H_{j}=\exp \left(\sum_{d=1}^{\infty} \frac{t^{d} P_{d}}{d}\right)
$$

These remarks reduce the proof of Proposition 1 to Proposition 2.

\subsection{Proof of Proposition 2.}

1.3.1. Strategy. We will prove Proposition 2 in two steps:

(i) We first reduce to special rational geometries via universality considerations.

(ii) A geometric argument using the moduli space of stable pairs will be given for rational surfaces $X$ with a sufficiently positive line bundle $M$.

1.3.2. Universality. Fix $\ell \geq 0$. We form the generating series

$Y_{X, M}^{(\ell)}=\sum_{B=\left(b_{1}, \ldots, b_{\ell}\right)} \frac{z_{1}^{b_{1}}}{b_{1} !} \cdots \frac{z_{\ell}^{b_{\ell}}}{b_{\ell} !} \sum_{n \geq 0} \sum_{a \geq 0} q^{n} t^{a} \cdot \int_{X^{[n]}} c_{n-a}\left(\left(M^{[n]}\right)^{\vee}\right) c\left(T X^{[n]}\right) \frac{P\left(\left(M^{[n]}\right)^{\vee}, B\right)}{c\left(\left(M^{[n]}\right)^{\vee}\right)}$.

The above expression is multiplicative in the sense that if $X=X_{1} \sqcup X_{2}$, then

$$
Y_{X, M}^{(\ell)}=Y_{X_{1}, M_{1}}^{(\ell)} \cdot Y_{X_{2}, M_{2}}^{(\ell)},
$$


where $M_{1}, M_{2}$ are the restrictions of $M$ to $X_{1}, X_{2}$ respectively. Claim (8) is a consequence of the following observations

$$
\begin{gathered}
X^{[n]}=\bigsqcup_{n_{1}+n_{2}=n} X_{1}^{\left[n_{1}\right]} \times X_{2}^{\left[n_{2}\right]} \\
M^{[n]}=\bigsqcup_{n_{1}+n_{2}=n} M_{1}^{\left[n_{1}\right]} \boxplus M_{2}^{\left[n_{2}\right]} \\
P_{i}\left(\left(M^{[n]}\right)^{\vee}\right)=\bigsqcup_{n_{1}+n_{2}=n} P_{i}\left(\left(M_{1}^{\left[n_{1}\right]}\right)^{\vee}\right)+P_{i}\left(\left(M_{2}^{\left[n_{2}\right]}\right)^{\vee}\right) .
\end{gathered}
$$

The factorials in the definition of $Y_{X, M}^{(\ell)}$ are engineered to offset the prefactors appearing in the binomial expansion $P_{i}^{b_{i}}$ of the third identity above.

As a consequence of above multiplicativity and the arguments of [EGL, we have

$$
Y_{X, M}^{(\ell)}=A_{1}^{K_{X}^{2}} \cdot A_{2}^{\chi\left(\mathcal{O}_{X}\right)} \cdot A_{3}^{M \cdot K_{X}} \cdot A_{4}^{M^{2}},
$$

for universal series $A_{1}, A_{2}, A_{3}, A_{4}$ in the variables $q, t, z_{1}, \ldots, z_{\ell}$. To prove Proposition 2 , we must show that

$$
\text { Coefficient of } t^{a} z_{1}^{b_{1}} \cdots z_{\ell}^{b_{\ell}} \text { in } A_{1}^{K_{X}^{2}} \cdot A_{2}^{\chi\left(\mathcal{O}_{X}\right)} \cdot A_{3}^{M \cdot K_{X}} \cdot A_{4}^{M^{2}}
$$

is a rational function in $q$.

Our method is to study special geometries $(X, M)$. Several choices are possible heres, for instance we could pick

(a) $X$ is the blowup of $\mathbb{P}^{2}$ at 1 point and $M=d H-e E$,

(b) $X$ is the blowup of $\mathbb{P}^{2}$ at 2 points and $M=d H-e_{1} E_{1}-e_{2} E_{2}$.

For the arguments of the following subsection, we will require $M$ sufficiently positive. For a concrete discussion, the results of $[\mathrm{R}]$ are useful. Specifically, if $\kappa$ is a fixed integer, a line bundle $M$, assumed not to equal a multiple of $\left(-K_{X}\right)$, is $\kappa$-very ample provided that the following inequalities hold

$$
\begin{aligned}
& \left(\mathrm{a}^{\prime}\right) d \geq e+\kappa, \quad e \geq \kappa, \\
& \left(\mathrm{b}^{\prime}\right) d \geq e_{1}+e_{2}+\kappa, \quad e_{1} \geq \kappa, \quad e_{2} \geq \kappa .
\end{aligned}
$$

We will furthermore assume 6

(c) there exists a divisor $L$ on $X$ such that $L \cdot M=1$.

Such an $L$ can be chosen in the form

$$
L=d^{\prime} H-e^{\prime} E \text { or } L=d^{\prime} H-e_{1}^{\prime} E_{1}-e_{2}^{\prime} E_{2}^{\prime}
$$

provided

\footnotetext{
${ }^{5}$ The simplest geometry $X=\mathbb{P}^{2}$ places numerical restrictions leading, at least a priori, to less precise results regarding the denominators of the answers.

${ }^{6}$ In the absence of (c), we have less control on the denominators of the rational functions thus obtained.
} 
$\left(c^{\prime}\right) \operatorname{gcd}(d, e)=1$ and $\operatorname{gcd}\left(d, e_{1}, e_{2}\right)=1$.

To complete the proof of Proposition 2 for arbitrary geometries, we need the following result.

Lemma 1. Fix $\ell \geq 0$ and $\kappa>0$. Assume that for all $0 \leq a \leq \kappa$, and all nonnegative $b_{1}, \ldots, b_{\ell}$

Coefficient of $t^{a} z_{1}^{b_{1}} \cdots z_{\ell}^{b_{\ell}}$ in $A_{1}^{K_{X}^{2}} \cdot A_{2}^{\chi\left(\mathcal{O}_{X}\right)} \cdot A_{3}^{M \cdot K_{X}} \cdot A_{4}^{M^{2}}$

is a rational function in $q$ for $(X, M)$ as above. Then the same coefficients are rational in $q$ for all pairs $(X, M)$.

Proof. Examples (a) and (b) give the rationality of the relevant coefficients in the expressions

$$
A_{1}^{8} \cdot A_{2} \cdot A_{3}^{-3 d+e} \cdot A_{4}^{d^{2}-e^{2}} \text { and } A_{1}^{7} \cdot A_{2} \cdot A_{3}^{-3 d+e_{1}+e_{2}} \cdot A_{4}^{d^{2}-e_{1}^{2}-e_{2}^{2}} .
$$

By varying $d, e, e_{1}, e_{2}$ for sufficiently large values with respect to $\kappa$ subject to the conditions above, we can reconstruct $A_{1}, A_{2}, A_{3}, A_{4}$ and conclude that their corresponding coefficients are rational in $q$.

1.3.3. Special geometries. We verify here the hypotheses of Lemma 1 for pairs $(X, M)$ satisfying all conditions above. The argument however applies more generally for sufficiently positive line bundles $M \rightarrow X$.

To keep the notation simple, we assume $B=\emptyset$ throughout Section 1.3.3. Thus

$$
Z_{X, M}[a]=\sum_{n \geq 0}^{\infty} q^{n} \cdot \int_{X^{[n]}} c_{n-a}\left(\left(M^{[n]}\right)^{\vee}\right) \cdot c\left(T X^{[n]}\right) \cdot s\left(\left(M^{[n]}\right)^{\vee}\right),
$$

where $s$ denotes the Segre class. We will indicate how to proceed with the general case $B \neq \emptyset$ in Section 1.3.7.

We begin by representing the Chern class $c_{n-a}\left(M^{[n]}\right)$ by a natural geometric cycle. To this end, we pick a general linear system $|V|$ in $|M|$ satisfying the following two properties:

(i) $\operatorname{dim}|V|=a$,

(ii) the curves in $|V|$ are irreducible and reduced.

This can be achieved if the coefficient $d$ of the hyperplane class in $M$ is chosen sufficiently large. Specifically, by [KT, Proposition 5.1], the assumption (ii) is satisfied as soon as $M$ is $(2 a+1)$-very ample. We write

$$
\pi: \mathcal{C} \rightarrow|V|
$$


for the universal curve. When regarded as the base of $\pi$, we write $\mathcal{B}$ instead of $|V|$. Let

$$
\pi:(\mathcal{C} / \mathcal{B})^{[n]} \rightarrow \mathcal{B}
$$

denote the relative Hilbert scheme of points. For all $n$, the space $(\mathcal{C} / \mathcal{B})^{[n]}$ is a nonsingular projective variety of dimension

$$
\operatorname{dim}(\mathcal{C} / \mathcal{B})^{[n]}=n+a
$$

by [GS, Theorem 46]. The assertion uses the assumption that $M$ is sufficiently positive, in particular, we need $M$ to be $a$-very ample. Furthermore, we have a natural morphism

$$
j:(\mathcal{C} / \mathcal{B})^{[n]} \rightarrow X^{[n]} .
$$

Pick $s_{0}, \ldots, s_{a}$ a basis for $|V|$, viewed as sections of $M$. Each section $s$ of $M$ induces a tautological section $s^{[n]}$ of the bundle $M^{[n]}$ via restriction

$$
\xi \rightarrow s_{\xi}, \quad s_{\xi} \in H^{0}\left(M \otimes \mathcal{O}_{\xi}\right)=\left.M^{[n]}\right|_{\xi} .
$$

Here $\xi \subset X$ is a length $n$ subscheme of $X$. We therefore obtain sections

$$
s_{0}^{[n]}, \ldots, s_{a}^{[n]}
$$

of $M^{[n]} \rightarrow X^{[n]}$. The degeneracy locus of these sections consists of subschemes $\xi$ of $X$ such that

$$
\xi \subset \mathcal{C}_{b}
$$

for some curve $\mathcal{C}_{b}$ of the linear system $|V|$. We therefore conclude

$$
j_{\star}(\mathcal{C} / \mathcal{B})^{[n]}=c_{n-a}\left(M^{[n]}\right) \cap\left[X^{[n]}\right] .
$$

We can rewrite (9) using equality (10) as

$$
\begin{aligned}
Z_{X, M}[a] & =\sum_{n=0}^{\infty} q^{n} \cdot \int_{X[n]} c_{n-a}\left(\left(M^{[n]}\right)^{\vee}\right) \cdot c\left(T X^{[n]}\right) \cdot s\left(\left(M^{[n]}\right)^{\vee}\right) \\
& =\sum_{n=0}^{\infty} q^{n}(-1)^{n-a} \int_{(\mathcal{C} / \mathcal{B})^{[n]}} j^{\star} c\left(T X^{[n]}\right) \cdot j^{\star} s\left(\left(M^{[n]}\right)^{\vee}\right) \\
& =(-1)^{a} Z_{\mathcal{C} / \mathcal{B}, M}(-q),
\end{aligned}
$$

where we define

$$
Z_{\mathcal{C} / \mathcal{B}, M}(q)=\sum_{n=0}^{\infty} q^{n} \int_{(\mathcal{C} / \mathcal{B})^{[n]}} j^{\star} c\left(T X^{[n]}-\left(M^{[n]}\right)^{\vee}\right) .
$$

We prove the rationality of $Z_{\mathcal{C} / \mathcal{B}, M}$. The key step is to show that the generating series encodes expressions of the form

$$
(-1)^{n}\left(p_{1}(n)+2^{n} \cdot p_{2}(n)\right) \text { for polynomials } p_{1}, p_{2} \text {. }
$$


Series of the form

$$
\sum_{n=0}^{\infty}(-1)^{n}\left(p_{1}(n)+2^{n} \cdot p_{2}(n)\right) q^{n}
$$

are rational functions in $q .7$ Hence, we will deduce Proposition 2 from the following result.

Lemma 2. For sufficiently positive line bundles $M \rightarrow X$ satisfying conditions $\left(a^{\prime}\right),\left(b^{\prime}\right)$, and $\left(c^{\prime}\right)$, and families of curves $\mathcal{C} \rightarrow \mathcal{B}$ satisfying (i) and (ii), the expression

$$
\int_{(\mathcal{C} / \mathcal{B})^{[n]}} j^{\star} c\left(T X^{[n]}-\left(M^{[n]}\right)^{\vee}\right)
$$

is of the form (因) for polynomials $p_{1}(n)$ and $p_{2}(n)$.

1.3.4. Proof of Lemma 国. We let $\mathcal{H} \rightarrow \mathcal{C}$ denote a relatively ample line bundle for the family

$$
\pi: \mathcal{C} \rightarrow \mathcal{B}
$$

For instance, we may pick

$$
\mathcal{H}=j^{\star} L
$$

for the line bundle $L$ whose existence was assumed in (c). Then, $\mathcal{H}$ has fiber degree 1 .

The following structures will play an important role in the proof of Lemma 2:

(i) the relative moduli space $\mathfrak{M} \rightarrow \mathcal{B}$ of torsion free rank 1 sheaves of degree 0 over the fibers of $\pi: \mathcal{C} \rightarrow \mathcal{B}$,

(ii) the universal sheaf

$$
\mathcal{J} \rightarrow \mathfrak{M} \times{ }_{\mathcal{B}} \mathcal{C}
$$

constructed in $\mathrm{AK}$ for families of reduced irreducible curves,

(iii) the universal subscheme

$$
\mathcal{Z}_{n} \hookrightarrow(\mathcal{C} / \mathcal{B})^{[n]} \times{ }_{\mathcal{B}} \mathcal{C},
$$

(iv) the universal subscheme $\mathcal{W}_{n}$ of $X^{[n]} \times X$.

We write

$$
\widehat{\pi}: \mathfrak{M} \times{ }_{\mathcal{B}} \mathcal{C} \rightarrow \mathfrak{M}
$$

for the base change of $\pi: \mathcal{C} \rightarrow \mathcal{B}$. We consider the sheaves

$$
\mathcal{J}, \mathcal{H} \rightarrow \mathfrak{M} \times{ }_{\mathcal{B}} \mathcal{C}
$$

where pullback from $\mathcal{C}$ is understood for the second line bundle. We set

$$
p_{n}: \mathbb{P}_{n}=\mathbb{P}\left(\widehat{\pi}_{\star}\left(\mathcal{J} \otimes \mathcal{H}^{n}\right)\right) \rightarrow \mathfrak{M} .
$$

\footnotetext{
${ }^{7}$ As a consequence, the denominators of the series of Euler characteristics (3) are products of $1-q$ and $1-2 q$ with various exponents. The same assertion holds true for the descendent series of Theorem 3. The example of Subsection 3.2.4 with $\beta=0$ also has the same denominators.
} 
For $n$ sufficiently large, $\mathbb{P}_{n}$ has fibers of constant dimension (by cohomology vanishing), so $\mathbb{P}_{n}$ a projective bundle over $\mathfrak{M}$. We write

$$
\zeta_{n}=\mathcal{O}_{\mathbb{P}_{n}}(1) .
$$

We will regard the relative Hilbert scheme $(\mathcal{C} / \mathcal{B})^{[n]}$ as a (subspace of the) moduli space $\mathcal{P}$ of stable pairs

$$
\left(F, s: \mathcal{O}_{X} \rightarrow F\right)
$$

on $X$ as explained in [PT2, Proposition B8]. Here,

$$
c_{1}(F)=c_{1}(M), \quad \chi(F)=1-g+n,
$$

with $g$ denoting the arithmetic genus of the linear series $|M|$. We furthermore require that the support of $F$ be contained in $\mathcal{B}=|V|$. The correspondence between the relative Hilbert scheme and stable pairs can be summarized as follows. For each subscheme

$$
\xi \subset \mathcal{C}_{b}
$$

the canonical sequence

$$
0 \rightarrow I_{\xi} \rightarrow \mathcal{O}_{\mathcal{C}_{b}} \rightarrow \mathcal{O}_{\xi} \rightarrow 0
$$

dualizes to

$$
0 \rightarrow \mathcal{O}_{\mathcal{C}_{b}} \rightarrow \operatorname{Hom}_{\mathcal{C}_{b}}\left(I_{\xi}, \mathcal{O}\right) \rightarrow \mathcal{E} x t_{\mathcal{C}_{b}}^{1}\left(\mathcal{O}_{\xi}, \mathcal{O}\right) \rightarrow 0
$$

where the last term has dimension zero and length $n$. Setting

$$
F=I_{\xi}^{\vee}=\operatorname{Hom}_{\mathcal{C}_{b}}\left(I_{\xi}, \mathcal{O}\right),
$$

we obtain a stable pair

$$
s: \mathcal{O}_{X} \rightarrow F
$$

on $X$ with the stated numerical invariants. By a result of [PT2],

$$
\operatorname{Ext}_{\mathcal{C}_{b}}^{\geq 1}\left(I_{\xi}, \mathcal{O}\right)=0 .
$$

Hence, the above dual can be interpreted as $\operatorname{RHom}_{\mathcal{C}_{b}}\left(I_{\xi}, \mathcal{O}\right)$ in the derived category.

As a consequence of the above identifications, there is a natural morphism

$$
\tau_{n}:(\mathcal{C} / \mathcal{B})^{[n]} \rightarrow \mathbb{P}_{n},
$$

Indeed, for the moduli space of stable pairs, we have a natural morphism

$$
\mathcal{P} \rightarrow \mathfrak{M}, \quad\left(F, s: \mathcal{O}_{X} \rightarrow F\right) \mapsto F \otimes \mathcal{H}^{-n} .
$$

We used here that $\mathcal{H}$ has fiber degree 1 , so that the twist $F \otimes \mathcal{H}^{-n}$ has fiber degree 0 . The fiber of the morphism (13) over a sheaf $J \in \mathfrak{M}$ is

$$
\mathbb{P} H^{0}\left(J \otimes \mathcal{H}^{n}\right) \text {. }
$$

The universal structure

$$
\mathcal{Z}_{n} \hookrightarrow(\mathcal{C} / \mathcal{B})^{[n]} \times{ }_{\mathcal{B}} \mathcal{C} \rightarrow \mathbb{P}_{n} \times{ }_{\mathcal{B}} \mathcal{C} \rightarrow \mathfrak{M} \times{ }_{\mathcal{B}} \mathcal{C}
$$


satisfies

$$
\mathcal{I}_{\mathcal{Z}_{n}}^{\vee}=\mathcal{J} \otimes \mathcal{H}^{n} \otimes \zeta_{n}
$$

In the above, duals are interpreted in the derived category.

We now examine the integrand which appears in Lemma 2. The following tautological structures over $\mathfrak{M}$ will be needed in the analysis.

(A) Consider the diagram

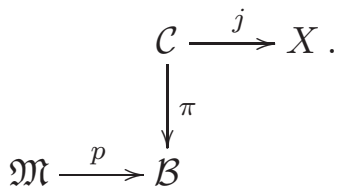

For a bundle $W \rightarrow X$, we define

$$
\bar{W}=p^{\star} \mathbf{R} \pi_{\star} j^{\star} W \rightarrow \mathfrak{M} .
$$

(B) Consider the diagram

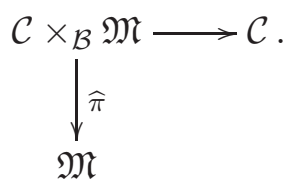

For a bundle $\mathcal{V} \rightarrow \mathcal{C}$, we set

$$
\begin{aligned}
& \mathcal{V}_{n} \rightarrow \mathfrak{M}, \quad \mathcal{V}_{n}=\mathbf{R} \widehat{\pi}_{\star}\left(\mathcal{V} \otimes \mathcal{J}^{\vee} \otimes \mathcal{H}^{-n}\right), \\
& \mathcal{V}_{n}^{\prime} \rightarrow \mathfrak{M}, \quad \mathcal{V}_{n}^{\prime}=\operatorname{Ext}_{\widehat{\pi}}^{\bullet}\left(\mathcal{J}^{\vee} \otimes \mathcal{H}^{-n}, \mathcal{V}\right), \\
& \mathcal{V}^{+} \rightarrow \mathfrak{M}, \quad \mathcal{V}^{+}=\operatorname{Ext}_{\widehat{\pi}}^{\bullet}\left(\mathcal{J}^{\vee}, \mathcal{V} \otimes \mathcal{J}^{\vee}\right)
\end{aligned}
$$

Pullbacks from the factors were suppressed in the expressions above. In particular, the above constructions make sense and will be used for bundles $\mathcal{V}$ pulled back from $X$.

By relative duality, we have

$$
\begin{aligned}
\mathcal{V}_{n}^{\prime} & =\operatorname{Ext}_{\widehat{\pi}}^{\bullet}\left(\mathcal{J}^{\vee} \otimes \mathcal{H}^{-n}, \mathcal{V}\right) \\
& =\operatorname{Ext}_{\widehat{\pi}}^{\bullet}\left(\mathcal{V}, \mathcal{J}^{\vee} \otimes \mathcal{H}^{-n} \otimes \omega_{\mathcal{C} / \mathcal{B}}\right)^{\vee}[1] \\
& =\mathbf{R} \widehat{\pi}_{\star}\left(\mathcal{J}^{\vee} \otimes \mathcal{H}^{-n} \otimes \mathcal{V}^{\vee} \otimes \omega_{\mathcal{C} / \mathcal{B}}\right)^{\vee}[1] \\
& =\left(\mathcal{V}^{\vee} \otimes \omega_{\mathcal{C} / \mathcal{B}}\right)_{n}^{\vee}[1] .
\end{aligned}
$$

The above constructions make sense for $K$-theory classes $\mathcal{V}$ as well.

Returning to Lemma 2, we now compute the pullbacks of the various tautological structures under the morphism

$$
j:(\mathcal{C} / \mathcal{B})^{[n]} \rightarrow X^{[n]} .
$$


Lemma 3. There are $K$-theory classes $\alpha, \beta$ on $\mathcal{C}$ and $\gamma$ on $\mathfrak{M}$ for which

$$
j^{\star}\left(T X^{[n]}-\left(M^{[n]}\right)^{\vee}\right)=\gamma+\alpha_{n} \cdot \zeta_{n}^{-1}+\left(\beta_{n}\right)^{\vee} \cdot \zeta_{n}
$$

over $(\mathcal{C} / \mathcal{B})^{[n]} \rightarrow \mathbb{P}_{n} \rightarrow \mathfrak{M}$. Furthermore, $\alpha$ has rank -1 and $\beta$ has rank 0 .

Proof. We compute the two pullbacks separately.

(i) First, recall

$$
M^{[n]}=\mathbf{R p r}_{\star}\left(M \otimes \mathcal{O}_{\mathcal{W}_{n}}\right)
$$

where $\mathcal{W}_{n}$ denotes the universal subscheme on $X^{[n]} \times X$ and

$$
\operatorname{pr}: X^{[n]} \times X \rightarrow X^{[n]} .
$$

The pullbacks on $M$ are omitted.

The pullback under $j$ is computed via the fibers of

$$
\pi:(\mathcal{C} / \mathcal{B})^{[n]} \times{ }_{\mathcal{B}} \mathcal{C} \rightarrow(\mathcal{C} / \mathcal{B})^{[n]} .
$$

We find

$$
j^{\star} M^{[n]}=\mathbf{R} \pi_{\star}\left(M \otimes \mathcal{O}_{\mathcal{Z}_{n}}\right) .
$$

Writing in $K$-theory

$$
\mathcal{O}_{\mathcal{Z}_{n}}=\mathcal{O}-\mathcal{I}_{\mathcal{Z}_{n}}=\mathcal{O}-\mathcal{J}^{\vee} \cdot \mathcal{H}^{-n} \cdot \zeta_{n}^{-1}
$$

via equation (14), we obtain

$$
j^{\star} M^{[n]}=\bar{M}-M_{n} \cdot \zeta_{n}^{-1} .
$$

Here, we have used the notations introduced in (A) and (B) above applied to the line bundle $M \rightarrow \mathcal{C} \rightarrow X$.

(ii) We now turn to $j^{\star} T X^{[n]}$. The alternating sum

$$
\mathcal{O}^{[n]}-T X^{[n]}+\left(\left(K_{X}\right)^{[n]}\right)^{\vee}
$$

computes fiber by fiber the complex

$$
\operatorname{Ext}^{0}\left(\mathcal{O}_{W}, \mathcal{O}_{W}\right)-\operatorname{Ext}^{1}\left(\mathcal{O}_{W}, \mathcal{O}_{W}\right)+\operatorname{Ext}^{2}\left(\mathcal{O}_{W}, \mathcal{O}_{W}\right)
$$

for subschemes $W$ of $X$. In families,

$$
j^{\star}\left(\mathcal{O}^{[n]}-T X^{[n]}+\left(\left(K_{X}\right)^{[n]}\right)^{\vee}\right)=j^{\star} \operatorname{Ext}_{X}^{\bullet}\left(\mathcal{O}_{\mathcal{W}_{n}}, \mathcal{O}_{\mathcal{W}_{n}}\right)
$$

where the subscript $X$ indicates the relative Ext's over the projection

$$
\operatorname{pr}: X^{[n]} \times X \rightarrow X^{[n]} \text {. }
$$


We seek to relate the relative $\operatorname{Ext}_{X}^{\bullet}$ and $\operatorname{Ext}_{\mathcal{C} / \mathcal{B}}^{\bullet}$ where the second Ext is computed via the projection

$$
\pi:(\mathcal{C} / \mathcal{B})^{[n]} \times{ }_{\mathcal{B}} \mathcal{C} \rightarrow(\mathcal{C} / \mathcal{B})^{[n]} .
$$

The key identity is

$$
j^{\star} \operatorname{Ext}_{X}^{\bullet}\left(\mathcal{O}_{\mathcal{W}_{n}}, \mathcal{O}_{\mathcal{W}_{n}}\right)=\operatorname{Ext}_{\mathcal{C} / \mathcal{B}}^{\bullet}\left(\mathcal{O}_{\mathcal{Z}_{n}}, \mathcal{O}_{\mathcal{Z}_{n}}\right)-\operatorname{Ext}_{\mathcal{C} / \mathcal{B}}^{\bullet}\left(\mathcal{O}_{\mathcal{Z}_{n}} \otimes \mathcal{N}, \mathcal{O}_{\mathcal{Z}_{n}}\right)
$$

Here, $\mathcal{N}$ is the conormal bundle of the inclusion

$$
\iota: \mathcal{C} \hookrightarrow \mathcal{B} \times X
$$

so that

$$
0 \rightarrow \mathcal{N} \rightarrow \iota^{\star} \Omega_{\mathcal{B} \times X / \mathcal{B}} \rightarrow \Omega_{\mathcal{C} / \mathcal{B}} \rightarrow 0
$$

or equivalently

$$
0 \rightarrow \mathcal{N} \rightarrow j^{\star} \Omega_{X} \rightarrow \Omega_{\mathcal{C} / \mathcal{B}} \rightarrow 0 .
$$

Equation (18) is the relative analogue of [T, Lemma 3.42] which gives the exactness of the sequence

$$
\operatorname{Ext}_{\mathcal{C}_{b}}^{i}\left(\mathcal{O}_{\xi}, \mathcal{O}_{\xi}\right) \rightarrow \operatorname{Ext}_{X}^{i}\left(\mathcal{O}_{\xi}, \mathcal{O}_{\xi}\right) \rightarrow \operatorname{Ext}_{\mathcal{C}_{b}}^{i-1}\left(\mathcal{O}_{\xi} \otimes \mathcal{N}, \mathcal{O}_{\xi}\right) \rightarrow \operatorname{Ext}_{\mathcal{C}_{b}}^{i+1}\left(\mathcal{O}_{\xi}, \mathcal{O}_{\xi}\right) \rightarrow \ldots
$$

for subschemes $\xi \subset \mathcal{C}_{b}$. To apply [T], we observe that $\left.\mathcal{N}\right|_{\mathcal{C}_{b}}$ is the conormal bundle of $\mathcal{C}_{b} \hookrightarrow X$, which follows by restricting the defining exact sequence to $\mathcal{C}_{b} .8$

With (18) understood, and by invoking (17), we find

$$
j^{\star} T X^{[n]}=j^{\star} \mathcal{O}^{[n]}+j^{\star}\left(\left(K_{X}\right)^{[n]}\right)^{\vee}-\operatorname{Ext}_{\mathcal{C} / \mathcal{B}}^{\bullet}\left(\mathcal{O}_{\mathcal{Z}_{n}}, \mathcal{O}_{\mathcal{Z}_{n}}\right)+\operatorname{Ext}_{\mathcal{C} / \mathcal{B}}^{\bullet}\left(\mathcal{O}_{\mathcal{Z}_{n}} \otimes \mathcal{N}, \mathcal{O}_{\mathcal{Z}_{n}}\right)
$$

The calculations in (i), specifically (16), yield the first two terms above

$$
j^{\star} \mathcal{O}^{[n]}=\overline{\mathcal{O}}-\mathcal{O}_{n} \cdot \zeta_{n}^{-1}
$$

and

$$
j^{\star}\left(\left(K_{X}\right)^{[n]}\right)^{\vee}={\overline{K_{X}}}^{\vee}-\left(\left(K_{X}\right)_{n}\right)^{\vee} \cdot \zeta_{n} .
$$

We examine the last two terms in (19). Substituting

$$
\mathcal{O}_{\mathcal{Z}_{n}}=\mathcal{O}-\mathcal{J}^{\vee} \mathcal{H}^{-n} \zeta_{n}^{-1}
$$

\footnotetext{
${ }^{8}$ We have

$$
\left.\left.0 \rightarrow \mathcal{T}_{\operatorname{or}_{\mathcal{C}}^{1}}^{1}\left(\Omega_{\mathcal{C} / \mathcal{B}}, \mathcal{O}_{\mathcal{C}_{b}}\right) \rightarrow \mathcal{N}\right|_{\mathcal{C}_{b}} \rightarrow \Omega_{X}\right|_{\mathcal{C}_{b}} \rightarrow \Omega_{\mathcal{C}_{b}} \rightarrow 0
$$

$\mathcal{T}_{\text {or }}{ }^{1}$ is supported on the finitely many singularities of $\mathcal{C}_{b}$. Since $\left.\mathcal{N}\right|_{\mathcal{C}_{b}}$ is locally free, $\mathcal{T}_{o r}{ }^{1}$ vanishes. Therefore, $\left.\mathcal{N}\right|_{\mathcal{C}_{b}}$ is the conormal bundle.
} 
yields

$$
\begin{aligned}
\operatorname{Ext}_{\mathcal{C} / \mathcal{B}}^{\bullet}\left(\mathcal{O}_{\mathcal{Z}_{n}}, \mathcal{O}_{\mathcal{Z}_{n}}\right)= & \operatorname{Ext}_{\mathcal{C} / \mathcal{B}}^{\bullet}\left(\mathcal{O}-\mathcal{J}^{\vee} \mathcal{H}^{-n} \zeta_{n}^{-1}, \mathcal{O}-\mathcal{J}^{\vee} \mathcal{H}^{-n} \zeta_{n}^{-1}\right) \\
= & \overline{\mathcal{O}}+\operatorname{Ext}_{\mathcal{C} / \mathcal{B}}^{\bullet}\left(\mathcal{J}^{\vee}, \mathcal{J}^{\vee}\right)-\mathbf{R} \pi_{\star}\left(\mathcal{J}^{\vee} \otimes \mathcal{H}^{-n}\right) \cdot \zeta_{n}^{-1} \\
& \quad-\operatorname{Ext}_{\mathcal{C} / \mathcal{B}}^{\bullet}\left(\mathcal{J}^{\vee} \otimes \mathcal{H}^{-n}, \mathcal{O}\right) \cdot \zeta_{n} \\
= & \overline{\mathcal{O}}+\mathcal{O}^{+}-\mathcal{O}_{n} \cdot \zeta_{n}^{-1}-\mathcal{O}_{n}^{\prime} \cdot \zeta_{n} .
\end{aligned}
$$

An entirely similar calculation shows that

$$
\operatorname{Ext}_{\mathcal{C} / \mathcal{B}}^{\bullet}\left(\mathcal{O}_{\mathcal{Z}_{n}} \otimes \mathcal{N}, \mathcal{O}_{\mathcal{Z}_{n}}\right)=\overline{\mathcal{N}^{\vee}}+\left(\mathcal{N}^{\vee}\right)^{+}-\left(\mathcal{N}^{\vee}\right)_{n} \cdot \zeta_{n}^{-1}-\left(\mathcal{N}^{\vee}\right)_{n}^{\prime} \cdot \zeta_{n}
$$

Collecting the last four equations into (19) we find

$j^{\star} T X^{[n]}=\left({\overline{K_{X}}}^{\vee}+\overline{\mathcal{N}}^{\vee}+\left(\mathcal{N}^{\vee}\right)^{+}-\mathcal{O}^{+}\right)-\left(\mathcal{N}^{\vee}\right)_{n} \cdot \zeta_{n}^{-1}+\left(\mathcal{O}_{n}^{\prime}-\left(\mathcal{N}^{\vee}\right)_{n}^{\prime}-\left(\left(K_{X}\right)_{n}\right)^{\vee}\right) \cdot \zeta_{n}$.

From (i) and (ii), we find that

$$
\begin{aligned}
j^{\star}\left(T X^{[n]}-\left(M^{[n]}\right)^{\vee}\right)=\left({\overline{K_{X}}}^{\vee}\right. & \left.+\overline{\mathcal{N}}^{\vee}-\bar{M}^{\vee}+\left(\mathcal{N}^{\vee}\right)^{+}-\mathcal{O}^{+}\right)-\left(\mathcal{N}^{\vee}\right)_{n} \cdot \zeta_{n}^{-1} \\
& +\left(\mathcal{O}_{n}^{\prime}-\left(\mathcal{N}^{\vee}\right)_{n}^{\prime}+\left(M_{n}\right)^{\vee}-\left(\left(K_{X}\right)_{n}\right)^{\vee}\right) \cdot \zeta_{n} .
\end{aligned}
$$

Using relative duality (15) for the last terms, we rewrite the above answer as

$$
\begin{aligned}
\left({\overline{K_{X}}}^{\vee}\right. & \left.+\overline{\mathcal{N} \vee}-\bar{M}^{\vee}+\left(\mathcal{N}^{\vee}\right)^{+}-\mathcal{O}^{+}\right)-\left(\mathcal{N}^{\vee}\right)_{n} \cdot \zeta_{n}^{-1} \\
& +\left(-\left(\omega_{\mathcal{C} / \mathcal{B}}\right)_{n}^{\vee}+\left(\mathcal{N} \otimes \omega_{\mathcal{C} / \mathcal{B}}\right)_{n}^{\vee}+\left(M_{n}\right)^{\vee}-\left(\left(K_{X}\right)_{n}\right)^{\vee}\right) \cdot \zeta_{n},
\end{aligned}
$$

which establishes Lemma 3 .

We return now to the proof of Lemma 2, First, we have

$$
\mathbb{P}_{n}=\mathbb{P}\left(\epsilon_{n}^{\prime}\right)
$$

where

$$
\epsilon_{n}^{\prime}=\mathbf{R} \widehat{\pi}_{\star}\left(\mathcal{J} \otimes \mathcal{H}^{n}\right)=\operatorname{Ext}_{\widehat{\pi}}^{\bullet}\left(\mathcal{J}^{\vee} \otimes \mathcal{H}^{-n}, \mathcal{O}\right)=\mathcal{O}_{n}^{\prime}
$$

in the notation (B) above. By Lemma 3 , expression (11) becomes

$$
\int_{\mathbb{P}_{n}} c\left(\gamma+\alpha_{n} \cdot \zeta_{n}^{-1}+\left(\beta_{n}\right)^{\vee} \cdot \zeta_{n}\right)
$$

which we will prove is of the form (因) for sufficiently large $n$.

The classes $\alpha$ and $\beta$ have ranks -1 and 0 respectively. Therefore,

$$
\operatorname{rank} \alpha_{n}=n+r_{1}, \operatorname{rank} \beta_{n}=r_{2}, \operatorname{rank} \epsilon_{n}^{\prime}=n+r_{3}+1,
$$

for constants $r_{1}, r_{2}, r_{3}$. Let $m$ denote the dimension of $\mathfrak{M}$, and let

$$
d=m+r_{3} .
$$


We obtain

$$
\int_{\mathbb{P}_{n}} c\left(\gamma+\alpha_{n} \cdot \zeta_{n}^{-1}+\left(\beta_{n}\right)^{\vee} \cdot \zeta_{n}\right)=\sum_{u+v+w=n+d} \int_{\mathbb{P}_{n}} c_{u}(\gamma) \cdot c_{v}\left(\alpha_{n} \cdot \zeta_{n}^{-1}\right) \cdot c_{w}\left(\left(\beta_{n}\right)^{\vee} \cdot \zeta_{n}\right) \text {. }
$$

The usual formulas give

$$
\begin{aligned}
& c_{v}\left(\alpha_{n} \cdot \zeta_{n}^{-1}\right)=\sum_{i=0}^{v}\left(\begin{array}{c}
\operatorname{rank} \alpha_{n}-i \\
v-i
\end{array}\right) \cdot c_{i}\left(\alpha_{n}\right) \cdot c_{1}\left(\zeta_{n}\right)^{v-i} \cdot(-1)^{v-i} \\
& c_{w}\left(\left(\beta_{n}\right)^{\vee} \cdot \zeta_{n}\right)=\sum_{j=0}^{w}\left(\begin{array}{c}
\operatorname{rank} \beta_{n}-j \\
w-j
\end{array}\right) \cdot c_{j}\left(\left(\beta_{n}\right)^{\vee}\right) \cdot c_{1}\left(\zeta_{n}\right)^{w-j} .
\end{aligned}
$$

We therefore are led to the expressions

$$
\sum_{u, v, w, i, j}(-1)^{v-i}\left(\begin{array}{c}
n+r_{1}-i \\
v-i
\end{array}\right)\left(\begin{array}{c}
r_{2}-j \\
w-j
\end{array}\right) \int_{\mathbb{P}_{n}} c_{u}(\gamma) \cdot c_{i}\left(\alpha_{n}\right) \cdot c_{j}\left(\left(\beta_{n}\right)^{\vee}\right) \cdot c_{1}\left(\zeta_{n}\right)^{v+w-i-j} .
$$

Integrating out $\zeta_{n}$ over the fibers of

$$
\mathbb{P}_{n} \rightarrow \mathfrak{M}
$$

we rewrite expressions (11) and (20) as

$$
\sum_{u+i+j+k=m} \sigma_{i j k}^{(n)} \int_{\mathfrak{M}} c_{u}(\gamma) \cdot c_{i}\left(\alpha_{n}\right) \cdot c_{j}\left(\left(\beta_{n}\right)^{\vee}\right) \cdot c_{k}\left(-\epsilon_{n}^{\prime}\right),
$$

where

$$
\sigma_{i j k}^{(n)}=\sum_{v+w=i+j+k+\left(n+r_{3}\right)}(-1)^{v-i}\left(\begin{array}{c}
n+r_{1}-i \\
v-i
\end{array}\right)\left(\begin{array}{c}
r_{2}-j \\
w-j
\end{array}\right) .
$$

The number of terms in this binomial sum could potentially grow with $n$. However, $i, j, k$ are bounded independently of $n$.

Lemma 4. For all $i, j, k, \sigma_{i j k}^{(n)}$ is of the form (因).

Lemma 5. There exists $M>0$ and $K$-classes $\mu_{0}^{(i)}, \ldots, \mu_{M}^{(i)}$ on $\mathfrak{M}$, for $1 \leq i \leq 3$, satisfying

$$
\begin{aligned}
\alpha_{n} & =\sum_{\ell=0}^{M} n^{\ell} \mu_{\ell}^{(1)}, \\
\left(\beta_{n}\right)^{\vee} & =\sum_{\ell=0}^{M} n^{\ell} \mu_{\ell}^{(2)}, \\
-\epsilon_{n}^{\prime} & =\sum_{\ell=0}^{M} n^{\ell} \mu_{\ell}^{(3)} .
\end{aligned}
$$


Assuming Lemmas 4 and 5, we return to (21) and the proof of Lemma 2, For any classes $\mu_{\ell}$ on $\mathfrak{M}$ with $0 \leq \ell \leq M$, we have

$$
c_{i}\left(\sum_{\ell=0}^{M} n^{\ell} \mu_{\ell}\right)=\left[\prod_{\ell=0}^{M}\left(1+c_{1}\left(\mu_{\ell}\right)+c_{2}\left(\mu_{\ell}\right)+\ldots\right)^{n^{\ell}}\right]_{(i)} .
$$

Furthermore,

$$
\left(1+c_{1}\left(\mu_{\ell}\right)+c_{2}\left(\mu_{\ell}\right)+\ldots\right)^{n^{\ell}}=\sum_{I} c_{I}\left(\mu_{\ell}\right)\left(\begin{array}{c}
n^{\ell} \\
I
\end{array}\right)
$$

where $I$ is a multi-index. Therefore

$$
c_{i}\left(\sum_{\ell=0}^{M} n^{\ell} \mu_{\ell}\right)=\sum c_{I_{0}}\left(\mu_{0}\right) c_{I_{1}}\left(\mu_{1}\right) \cdots c_{I_{M}}\left(\mu_{M}\right) \cdot\left(\begin{array}{c}
1 \\
I_{0}
\end{array}\right)\left(\begin{array}{c}
n \\
I_{1}
\end{array}\right) \cdots\left(\begin{array}{c}
n^{M} \\
I_{M}
\end{array}\right),
$$

where, for degree reasons,

$$
\left|I_{0}\right|+\ldots+\left|I_{M}\right|=i \text {. }
$$

Thus $I_{j}$ 's have entries bounded by $i \leq m$, and the above expression is therefore polynomial in $n$. As a result, (21) becomes

$$
\sum_{u+i+j+k=m} \sigma_{i j k}^{(n)} \int_{\mathfrak{M}} c_{u}(\gamma) \cdot c_{i}\left(\sum_{\ell=0}^{M} n^{\ell} \mu_{\ell}^{(1)}\right) \cdot c_{j}\left(\sum_{\ell=0}^{M} n^{\ell} \mu_{\ell}^{(2)}\right) \cdot c_{k}\left(\sum_{\ell=0}^{M} n^{\ell} \mu_{\ell}^{(3)}\right)
$$

which is of the form (因) by Lemma 4 and the above observations. The proof of Lemma 2 will therefore be complete once Lemmas 4 and 5 are proven.

1.3.5. Proof of Lemma 4. The notation

$$
v^{\text {new }}=v-i, \quad w^{\text {new }}=w-j, a=r_{1}-i, \quad b=r_{2}-j, c=r_{3}+k
$$

will be more convenient for us. With the new conventions, the expression in Lemma 3 becomes

$$
\sigma^{(n)}=\sum_{v+w=n+c}(-1)^{v}\left(\begin{array}{c}
n+a \\
v
\end{array}\right)\left(\begin{array}{c}
b \\
w
\end{array}\right)=\operatorname{Coeff}_{x^{n+c}}(1-x)^{n+a} \cdot(1+x)^{b} .
$$

We rewrite the above as a residue

$$
\sigma^{(n)}=\operatorname{Res}_{x=0} \frac{(1-x)^{n+a} \cdot(1+x)^{b}}{x^{n+c+1}} d x .
$$

We change variables

so that the differential form transforms to

$$
y=\frac{1-x}{x} \Longrightarrow x=\frac{1}{y+1}
$$

$$
\omega=-y^{n+a} \cdot(y+2)^{b} \cdot(y+1)^{e} d y,
$$

for some constant $e$. Thus

$$
\sigma^{(n)}=\operatorname{Res}_{y=\infty} \omega=-\operatorname{Res}_{y=-1} \omega-\operatorname{Res}_{y=-2} \omega,
$$


via the Residue Theorem. There are no poles for $\omega$ at $y=0$ for $n$ sufficiently large.

The residues at

$$
y=-1, y=-2
$$

correspond to the two terms of (因). Indeed, for $y=-1$, we have

$$
\begin{aligned}
\operatorname{Res}_{y=-1} y^{n+a} \cdot(y+2)^{b} \cdot(y+1)^{e} d y & =\operatorname{Res}_{z=0}(z-1)^{n+a} \cdot(z+1)^{b} \cdot z^{e} d z \\
& =\operatorname{Coeff}_{z^{-e-1}}(z-1)^{n+a} \cdot(z+1)^{b} \\
& =(-1)^{n} \sum_{v+w=-e-1}(-1)^{v+a}\left(\begin{array}{c}
n+a \\
v
\end{array}\right)\left(\begin{array}{c}
b \\
w
\end{array}\right) .
\end{aligned}
$$

The latter sum is finite, hence manifestly polynomial in $n$. A similar calculation shows that the residue at $y=-2$ is of the form

$$
(-2)^{n} \cdot \text { polynomial in } n \text {, }
$$

completing the proof.

1.3.6. Proof of Lemma國. We present the argument for $\alpha_{n}$. The proofs of the other two statements are the same. Consider the class

$$
x=\mathcal{H}-1
$$

viewed in the $K$-theory of $\mathcal{C}$. Since $\mathcal{C}$ is nonsingular and projective, the Chern character gives an isomorphism

$$
\operatorname{ch}: K(\mathcal{C}) \otimes \mathbb{Q} \rightarrow A^{\star}(\mathcal{C}) \otimes \mathbb{Q} .
$$

Clearly $\operatorname{ch}(x) \in A^{>0}(\mathcal{C})$, hence $\operatorname{ch}(x)^{M}=0$ for degree reasons, for some $M>0$. So

$$
x^{M}=0
$$

in $K$-theory, hence

$$
(\mathcal{H}-1)^{M}=0 .
$$

We conclude

$$
\sum_{i=0}^{M}(-1)^{i} \cdot\left(\begin{array}{c}
M \\
i
\end{array}\right) \cdot \mathcal{H}^{i}=0
$$

and therefore

$$
\sum_{i=0}^{M}(-1)^{i} \cdot\left(\begin{array}{c}
M \\
i
\end{array}\right) \cdot \mathcal{H}^{i-n} \cdot \mathcal{J}^{\vee} \alpha=0
$$

in $K\left(\mathfrak{M} \times{ }_{B} \mathcal{C}\right)$. Pushing forward via $\widehat{\pi}$ to $\mathfrak{M}$, we obtain

$$
\sum_{i=0}^{M}(-1)^{i} \cdot\left(\begin{array}{c}
M \\
i
\end{array}\right) \cdot \alpha_{n-i}=0 .
$$


This linear recursion in the $\alpha$ 's can be solved explicitly. Note that the characteristic equation

$$
\sum_{i=0}^{M}(-1)^{i} \cdot\left(\begin{array}{c}
M \\
i
\end{array}\right) \cdot r^{-i}=0
$$

has $M$ repeated roots all equal to 1 .

1.3.7. The case $B \neq \emptyset$. The last step of the proof of Proposition 2 is to treat the case when the sequence $B \neq \emptyset$.

The argument is similar to the $B=\emptyset$ case, but for completeness we indicate the main points. We wish to prove that

$$
Z_{X, M}[a, B]=\sum_{n=0}^{\infty} q^{n} \cdot \int_{X^{[n]}} c_{n-a}\left(\left(M^{[n]}\right)^{\vee}\right) \cdot c\left(T X^{[n]}\right) \cdot \frac{P\left(\left(M^{[n]}\right)^{\vee}, B\right)}{c\left(\left(M^{[n]}\right)^{\vee}\right)}
$$

is rational in $q$. Following the reasoning in Section 1.3.3 and using Lemma 3, it suffices to show

$$
\int_{\mathbb{P}_{n}} c\left(\gamma+\alpha_{n} \cdot \zeta_{n}^{-1}+\left(\beta_{n}\right)^{\vee} \cdot \zeta_{n}\right) \cdot P\left(j^{\star}\left(M^{[n]}\right)^{\vee}, B\right)
$$

is of the form (因), for $n$ large enough. This is analogous to Lemma 2 ,

By (16), we have

$$
P_{b}\left(j^{\star}\left(M^{[n]}\right)^{\vee}\right)=P_{b}\left(\bar{M}^{\vee}\right)+P_{b}\left((-M)_{n}^{\vee} \cdot \zeta_{n}\right),
$$

where we have extended the definition of $P_{b}$ given in (7) to $K$-theory by linearity. We multiply out the $P_{b}$ 's for the values of $b$ determined by the sequence $B$. Since $\bar{M}$ is a $K$-theory class on $\mathfrak{M}$, we can combine terms of the form $P_{b}\left(\bar{M}^{\vee}\right)$ and $c(\gamma)$ into a single cohomology class $\lambda$ over $\mathfrak{M}$. We are led to expressions of the form

$$
\int_{\mathbb{P}_{n}} \lambda \cdot c\left(\alpha_{n} \cdot \zeta_{n}^{-1}+\left(\beta_{n}\right)^{\vee} \cdot \zeta_{n}\right) \cdot P_{b_{1}}\left((-M)_{n}^{\vee} \cdot \zeta_{n}\right) \cdots P_{b_{m}}\left((-M)_{n}^{\vee} \cdot \zeta_{n}\right)
$$

for nonnegative integers $b_{1}, \ldots, b_{m}$.

Recall from Section 1.3 .4 that

$$
(-M)_{n}=-\mathbf{R} \widehat{\pi}_{\star}\left(M \otimes \mathcal{J}^{\vee} \otimes \mathcal{H}^{-n}\right) .
$$

By inspecting the fiber degree, we see that $(-M)_{n}$ is represented by a vector bundle for $n$ sufficiently large, and we write $x_{1}, \ldots, x_{r}$ for the Chern roots. The rank $r$ depends on $n$ linearly. We have

$$
P_{b}\left((-M)_{n}^{\vee} \cdot \zeta_{n}\right)=\sum_{i=1}^{r} \frac{1}{\left(1-x_{i}+c_{1}\left(\zeta_{n}\right)\right)^{b}}
$$


We expand

$$
\frac{1}{\left(1-x_{i}+c_{1}\left(\zeta_{n}\right)\right)^{b}}=\frac{1}{\left(1-x_{i}\right)^{b}} \cdot\left(1+\frac{c_{1}\left(\zeta_{n}\right)}{1-x_{i}}\right)^{-b}=\sum_{\ell=0}^{\infty}\left(\begin{array}{c}
-b \\
\ell
\end{array}\right) \cdot \frac{c_{1}\left(\zeta_{n}\right)^{\ell}}{\left(1-x_{i}\right)^{\ell+b}} .
$$

Thus

$$
P_{b}\left((-M)_{n}^{\vee} \cdot \zeta_{n}\right)=\sum_{\ell=0}^{\infty}\left(\begin{array}{c}
-b \\
\ell
\end{array}\right) \cdot c_{1}\left(\zeta_{n}\right)^{\ell} \cdot \mathrm{p}_{\ell+b}\left((-M)_{n}\right)
$$

where the classes $\mathrm{p}$ on $\mathfrak{M}$ have terms of mixed degrees. In fact, the p's are series in the Chern classes of the argument whose coefficients are independent of $n$. The only exception is the constant term which is linear in $n$ being equal to the rank.

Expression (22) becomes

$$
\begin{aligned}
\sum_{\ell_{1}, \cdots, \ell_{m}}\left(\begin{array}{c}
-b_{1} \\
\ell_{1}
\end{array}\right) \cdots\left(\begin{array}{c}
-b_{m} \\
\ell_{m}
\end{array}\right) \cdot \int_{\mathbb{P}_{n}} \lambda \cdot c\left(\alpha_{n} \cdot \zeta_{n}^{-1}+\left(\beta_{n}\right)^{\vee} \cdot \zeta_{n}\right) \cdot c_{1}\left(\zeta_{n}\right)^{\ell_{1}+\ldots+\ell_{m}} \\
\cdot \mathrm{p}_{\ell_{1}+b_{1}}\left((-M)_{n}\right) \cdots \mathrm{p}_{\ell_{m}+b_{m}}\left((-M)_{n}\right) .
\end{aligned}
$$

To go further, we apply the same reasoning that led to equation (21). Accounting for the extra p's and their prefactors, the above expression becomes

$$
\sum_{i, j, k, \vec{a}} \sigma_{i, j, k, \vec{a}}^{(n)} \int_{\mathfrak{M}} \lambda \cdot c_{i}\left(\alpha_{n}\right) \cdot c_{j}\left(\left(\beta_{n}\right)^{\vee}\right) \cdot c_{k}\left(-\epsilon_{n}^{\prime}\right) \cdot \mathrm{p}_{a_{1}}\left((-M)_{n}\right) \cdots \mathrm{p}_{a_{m}}\left((-M)_{n}\right)
$$

where

$$
\ell_{1}+b_{1}=a_{1}, \ldots, \ell_{m}+b_{m}=a_{m} .
$$

The prefactor here equals

$$
\sigma_{i, j, k, \vec{a}}^{(n)}=\sum_{v, w, \vec{b}, \vec{\ell}}\left(\begin{array}{c}
-b_{1} \\
\ell_{1}
\end{array}\right) \cdots\left(\begin{array}{c}
-b_{m} \\
\ell_{m}
\end{array}\right) \cdot(-1)^{v-i}\left(\begin{array}{c}
n+r_{1}-i \\
v-i
\end{array}\right)\left(\begin{array}{c}
r_{2}-j \\
w-j
\end{array}\right),
$$

where in the summation we have

$$
v+w+|\ell|=i+j+k+\left(n+r_{3}\right), \quad \vec{\ell}+\vec{b}=\vec{a} .
$$

Each integral over $\mathfrak{M}$ is polynomial in $n$. Indeed, dimension constraints select only finitely many homogeneous pieces from the p's and from $\lambda$, of bounded degree. We then argue by invoking Lemma 5 applied to

$$
\alpha_{n},\left(\beta_{n}\right)^{\vee},-\epsilon_{n}^{\prime},(-M)_{n}
$$

combined with the analysis that followed the statement of the Lemma. 
To conclude, it remains to prove $\sigma^{(n)}$ is of the form (因), the analogue of Lemma 4 . We have

$$
\begin{aligned}
\sigma_{i, j, k, \vec{a}}^{(n)} & =\sum_{v, w, \vec{b}, \vec{\ell}}\left(\begin{array}{c}
-b_{1} \\
\ell_{1}
\end{array}\right) \cdots\left(\begin{array}{c}
-b_{m} \\
\ell_{m}
\end{array}\right) \cdot(-1)^{v-i}\left(\begin{array}{c}
n+r_{1}-i \\
v-i
\end{array}\right)\left(\begin{array}{c}
r_{2}-j \\
w-j
\end{array}\right) \\
& =\sum_{v, w, \vec{b}, \vec{\ell}}\left(\begin{array}{c}
b_{1}+\ell_{1}-1 \\
\ell_{1}
\end{array}\right) \cdots\left(\begin{array}{c}
b_{m}+\ell_{m}-1 \\
\ell_{m}
\end{array}\right) \cdot(-1)^{v-i+|\ell|}\left(\begin{array}{c}
n+r_{1}-i \\
v-i
\end{array}\right)\left(\begin{array}{c}
r_{2}-j \\
w-j
\end{array}\right) \\
& =\sum_{v, w,|\ell|}(-1)^{v-i+|\ell|}\left(\begin{array}{c}
|a|-m \\
|\ell|
\end{array}\right)\left(\begin{array}{c}
n+r_{1}-i \\
v-i
\end{array}\right)\left(\begin{array}{c}
r_{2}-j \\
w-j
\end{array}\right)
\end{aligned}
$$

where the Vandermonde identity was used in the last line to sum over $\ell_{1}+\ldots+\ell_{m}=|\ell|$. Writing $|\ell|+v=v^{\prime}$, and using the Vandermonde identity one more time, we obtain

$$
\sigma_{i, j, k, \vec{a}}^{(n)}=\sum_{v^{\prime}+w=n+\mathrm{const}}(-1)^{v^{\prime}-i}\left(\begin{array}{c}
(|a|-m)+\left(n+r_{1}-i\right) \\
v^{\prime}-i
\end{array}\right)\left(\begin{array}{c}
r_{2}-j \\
w-j
\end{array}\right) .
$$

This is exactly the type of expression considered in Lemma 4. The proof of Proposition 2 is complete.

1.3.8. Example. We illustrate the methods used in the proof of Proposition 2 with the computation of the series

$$
Z=\sum_{n=1}^{\infty} q^{n} \int_{X^{[n]}} c_{n-1}\left(\left(M^{[n]}\right)^{\vee}\right) \cdot c\left(T X^{[n]}\right) \cdot s\left(\left(M^{[n]}\right)^{\vee}\right)
$$

in the special case

$$
X=B \times F \rightarrow B \text { and } M=\mathcal{O}_{B}(1),
$$

where $B=F=\mathbb{P}^{1}$. The family of curves in the fiber class $\mathcal{C} \rightarrow B$ is isomorphic to the surface $X \rightarrow B$, and the relative Hilbert scheme of points is the product

$$
(\mathcal{C} / B)^{[n]}=B \times \mathbb{P}^{n} .
$$

By (14), the universal subscheme

$$
\mathcal{Z}_{n} \hookrightarrow \mathcal{C} \times{ }_{B}(\mathcal{C} / B)^{[n]}=X \times \mathbb{P}^{n}
$$

satisfies

$$
I_{\mathcal{Z}_{n}}=\mathcal{O}_{F}(-n) \otimes \mathcal{O}_{\mathbb{P}^{n}}(-1)
$$

We represent

$$
c_{n-1}\left(M^{[n]}\right)=(\mathcal{C} / B)^{[n]}=\left[B \times \mathbb{P}^{n}\right]
$$

so that

$$
Z=\sum_{n=1}^{\infty} q^{n}(-1)^{n-1} \int_{B \times \mathbb{P}^{n}} j^{\star} c\left(T X^{[n]}-\left(M^{[n]}\right)^{\vee}\right) .
$$

Here, we continue to write

$$
j: B \times \mathbb{P}^{n} \rightarrow X^{[n]}
$$


for the natural morphism. Let

$$
\pi: X \times \mathbb{P}^{n} \rightarrow B \times \mathbb{P}^{n}
$$

denote the projection. We compute the tautological structures

$$
\begin{aligned}
j^{\star} M^{[n]} & =\mathbf{R} \pi_{\star}\left(M \otimes \mathcal{O}_{\mathcal{Z}_{n}}\right) \\
& =\mathbf{R} \pi_{\star}\left(M-M \otimes \mathcal{O}_{F}(-n) \otimes \mathcal{O}_{\mathbb{P}^{n}}(-1)\right) \\
& =\mathcal{O}_{B}(1)+\mathbb{C}^{n-1} \otimes \mathcal{O}_{B}(1) \otimes \mathcal{O}_{\mathbb{P}^{n}}(-1) .
\end{aligned}
$$

Similarly,

$$
\begin{aligned}
j^{\star} \mathcal{O}_{X}^{[n]} & =\mathcal{O}+\mathbb{C}^{n-1} \otimes \mathcal{O}_{\mathbb{P}^{n}}(-1) \\
j^{\star}\left(K_{X}\right)^{[n]} & =-\mathcal{O}_{B}(-2)+\mathbb{C}^{n+1} \otimes \mathcal{O}_{B}(-2) \otimes \mathcal{O}_{\mathbb{P}^{n}}(-1) .
\end{aligned}
$$

By (19), we have

$$
j^{\star} T X^{[n]}=j^{\star} \mathcal{O}_{X}^{[n]}+j^{\star}\left(\left(K_{X}\right)^{[n]}\right)^{\vee}-\operatorname{Ext}_{\mathcal{C} / B}^{\bullet}\left(\mathcal{O}_{Z_{n}}, \mathcal{O}_{Z_{n}}\right)+\operatorname{Ext}_{\mathcal{C} / B}^{\bullet}\left(\mathcal{O}_{Z_{n}}, \mathcal{O}_{Z_{n}}\right) \otimes \mathcal{O}_{B}(2) .
$$

Here, we have used

$$
\mathcal{N}=\Omega_{B}=\mathcal{O}_{B}(-2)
$$

Furthermore,

$$
\begin{aligned}
\operatorname{Ext}_{\mathcal{C} / B}^{\bullet}\left(\mathcal{O}_{Z_{n}}, \mathcal{O}_{Z_{n}}\right) & =\operatorname{Ext}_{\mathcal{C} / B}^{\bullet}\left(\mathcal{O}-\mathcal{O}_{F}(-n) \otimes \mathcal{O}_{\mathbb{P}^{n}}(-1), \mathcal{O}-\mathcal{O}_{F}(-n) \otimes \mathcal{O}_{\mathbb{P}^{n}}(-1)\right) \\
& =\mathbb{C}^{2} \otimes \mathcal{O}_{B}+\mathbb{C}^{n-1} \otimes \mathcal{O}_{\mathbb{P}^{n}}(-1)-\mathbb{C}^{n+1} \otimes \mathcal{O}_{\mathbb{P}^{n}}(1) .
\end{aligned}
$$

After substituting, we find

$$
\begin{aligned}
j^{\star}\left(T X^{[n]}-\left(M^{[n]}\right)^{\vee}\right)=\left(-\mathcal{O}_{B}-\right. & \left.\mathcal{O}_{B}(-1)+\mathcal{O}_{B}(2)\right)+\mathbb{C}^{n-1} \otimes \mathcal{O}_{B}(2) \otimes \mathcal{O}_{\mathbb{P}}(-1) \\
& +\mathbb{C}^{n+1} \otimes \mathcal{O}_{\mathbb{P}^{n}}(1)-\mathbb{C}^{n-1} \otimes \mathcal{O}_{B}(-1) \otimes \mathcal{O}_{\mathbb{P}}(1) .
\end{aligned}
$$

With $h, \zeta$ denoting the hyperplane classes on $B$ and $\mathbb{P}^{n}$, we arrive at the integral

$$
\int_{B \times \mathbb{P}^{n}} \frac{1+2 h}{1-h} \cdot(1+\zeta)^{n+1} \cdot\left(\frac{1-\zeta+2 h}{1+\zeta-h}\right)^{n-1} .
$$

The last expression equals

$$
\operatorname{Coeff}_{h \zeta^{n}} \frac{1+2 h}{1-h} \cdot(1+\zeta)^{n+1} \cdot\left(\frac{1-\zeta+2 h}{1+\zeta-h}\right)^{n-1}=(-1)^{n} \cdot(4 n-10) .
$$

Hence, we can write (24) as

$$
Z=\sum_{n=1}^{\infty} q^{n}(10-4 n)=\frac{q(6-10 q)}{(1-q)^{2}}
$$

For another example, if $X$ is a $K 3$ surface and $M=\mathcal{O}_{X}$, the series

$$
Z=\sum_{n=1}^{\infty} q^{n} \int_{X^{[n]}} c_{n-1}\left(\left(M^{[n]}\right)^{\vee}\right) \cdot c\left(T X^{[n]}\right) \cdot s\left(\left(M^{[n]}\right)^{\vee}\right)=\frac{24 q}{(1-q)^{2}}
$$


was computed in Proposition 40 of [OP1].

Evaluating (24) in closed form for all pairs $(X, M)$ is likely possible.

\section{Descendent series of Punctual Quot schemes: Theorem 2}

2.1. Overview. The goal here is to prove Theorem 2, Throughout Section 2, we set $\beta=0$. We will establish the rationality of the descendent series

$Z_{X, N}\left(\alpha_{1}, \ldots, \alpha_{\ell} \mid k_{1}, \ldots, k_{\ell}\right)=\sum_{n=0}^{\infty} q^{n} \cdot \int_{\left[\operatorname{Quot}_{X}\left(\mathbb{C}^{N}, n\right)\right]^{\mathrm{vir}}} \operatorname{ch}_{k_{1}}\left(\alpha_{1}^{[n]}\right) \cdots \operatorname{ch}_{k_{\ell}}\left(\alpha_{\ell}^{[n]}\right) c\left(T^{\mathrm{vir}}\right.$ Quot $)$.

Our argument follows the strategy of the proof of Theorem 18 of [OP1].

2.2. Proof of Theorem 2. We will explain shortly that for fixed rank $\alpha_{i}=r_{i}$, the series $Z_{X, N}\left(\alpha_{1}, \ldots, \alpha_{\ell} \mid k_{1}, \ldots, k_{\ell}\right)$ is given by universal expressions in the Chern classes of the $\alpha_{i}$ 's. Furthermore, for each $k_{1}, \ldots, k_{\ell}$, the series

$$
Z_{X, N}\left(\alpha_{1}, \ldots, \alpha_{\ell} \mid k_{1}, \ldots, k_{\ell}\right)
$$

is additive in $\alpha_{1}, \ldots, \alpha_{\ell}$ separately. Thus, invoking the splitting principle, it suffices to assume that

$$
\text { rank } \alpha_{i}=1 \text { for all } 1 \leq i \leq \ell .
$$

The proof below can also be directly written for $\alpha_{i}$ 's of arbitrary ranks, at the expense of more complicated notation.

Since the Chern character is polynomial in the Chern classes, we equivalently consider the series

$$
Z=\sum_{n=0}^{\infty} q^{n} \cdot \int_{\left[\operatorname{Quot}_{X}\left(\mathbb{C}^{N}, n\right)\right]^{\mathrm{vir}}} c_{k_{1}}\left(\alpha_{1}^{[n]}\right) \cdots c_{k_{\ell}}\left(\alpha_{\ell}^{[n]}\right) \cdot c\left(T^{\mathrm{vir}} \text { Quot }\right) .
$$

Let $x_{1}, \ldots, x_{\ell}$ be formal variables. Write

$$
c_{x}=1+x c_{1}+x^{2} c_{2}+\ldots
$$

for the total Chern class, and set

$$
W=\sum_{n=0}^{\infty} q^{n} \cdot \int_{\left[\operatorname{Quot}_{X}\left(\mathbb{C}^{N}, n\right)\right]^{\mathrm{vir}}} c_{x_{1}}\left(\alpha_{1}^{[n]}\right) \ldots c_{x_{\ell}}\left(\alpha_{\ell}^{[n]}\right) \cdot c\left(T^{\mathrm{vir}} \text { Quot }\right) .
$$

The series $Z$ is found by extracting the coefficient of $x_{1}^{k_{1}} \cdots x_{\ell}^{k_{\ell}}$ in $W$ :

$$
Z=\left.\frac{1}{k_{1} !} \cdots \frac{1}{k_{\ell} !} \cdot \frac{\partial^{k_{1}}}{\partial^{k_{1}} x_{1}} \cdots \frac{\partial^{k_{\ell}}}{\partial^{k_{\ell}} x_{\ell}} W\right|_{x_{1}=\ldots=x_{\ell}=0} .
$$

As in [OP1], we have a factorization

$$
W=\mathrm{A}^{K_{X}^{2}} \cdot \mathrm{B}^{\chi(X)} \cdot \prod_{i=1}^{\ell} \mathrm{C}_{i}^{c_{1}\left(\alpha_{i}\right) \cdot K_{X}} \cdot \mathrm{D}_{i}^{c_{1}\left(\alpha_{i}\right)^{2}} \cdot \mathrm{E}_{i}^{c_{2}\left(\alpha_{i}\right)} \cdot \prod_{1 \leq i<j \leq \ell} \mathrm{F}_{i j}^{c_{1}\left(\alpha_{i}\right) \cdot c_{1}\left(\alpha_{j}\right)}
$$


for universal series $\mathrm{A}, \mathrm{B}, \mathrm{C}_{i}, \mathrm{D}_{i}, \mathrm{E}_{i}, \mathrm{~F}_{i j}$ that depend on $q$ and $x_{m}$. We study the rationality of these series and of their $x_{m}$-derivatives.

To this end, we pick convenient geometries. Take a nonsingular projective surface $X$ which admits a nonsingular connected canonical curve

$$
\iota: C \hookrightarrow X
$$

of genus $g$. We move the calculation to the punctual Quot scheme of the curve $C$ :

$$
\iota: \operatorname{Quot}_{C}\left(\mathbb{C}^{N}, n\right) \rightarrow \operatorname{Quot}_{X}\left(\mathbb{C}^{N}, n\right) .
$$

By [OP1, Lemma 34], we have

$$
\iota_{\star}\left[\operatorname{Quot}_{C}\left(\mathbb{C}^{N}, n\right)\right]=(-1)^{n}\left[\operatorname{Quot}_{X}\left(\mathbb{C}^{N}, n\right)\right]^{\mathrm{vir}} .
$$

Furthermore, as remarked in equation (42) of [OP1], in $K$-theory we have the decomposition

$$
\iota^{\star} T^{\mathrm{vir}} \operatorname{Quot}_{X}\left(\mathbb{C}^{N}, n\right)=T \operatorname{Quot}_{C}\left(\mathbb{C}^{N}, n\right)+\mathcal{T}_{n} .
$$

Here, $\mathcal{T}_{n} \rightarrow \operatorname{Quot}_{C}\left(\mathbb{C}^{N}, n\right)$ is the virtual bundle given pointwise over the quotient

$$
\mathbb{C}^{N} \otimes \mathcal{O}_{C} \rightarrow Q
$$

by the expression

$$
\mathcal{T}_{n}=\operatorname{Ext}_{C}^{\bullet}(Q, Q \otimes \Theta),
$$

where $\Theta=N_{C / X}$ is the associated theta characteristic. As a consequence, we have

$$
W=\sum_{n=0}^{\infty} q^{n}(-1)^{n} \cdot \int_{\text {Quot }_{C}\left(\mathbb{C}^{N}, n\right)} c_{x_{1}}\left(\iota^{*} \alpha_{1}\right) \cdots c_{x_{\ell}}\left(\iota^{*} \alpha_{n}\right) \cdot c\left(\text { TQuot }_{C}\right) \cdot c\left(\mathcal{T}_{n}\right) .
$$

The above expression does not depend on the surface $X$, which we will ignore from now on. It follows then that

$$
\mathrm{B}=1, \quad \mathrm{D}_{i}=1, \quad \mathrm{E}_{i}=1, \quad \mathrm{~F}_{i j}=1 .
$$

Therefore, for $\beta_{i}=\iota^{\star} \alpha_{i}$, we have

$$
W=\sum_{n=0}^{\infty} q^{n}(-1)^{n} \cdot \int_{\operatorname{Quot}_{C}\left(\mathbb{C}^{N}, n\right)} c_{x_{1}}\left(\beta_{1}^{[n]}\right) \cdots c_{x_{\ell}}\left(\beta_{\ell}^{[n]}\right) \cdot c\left(\text { TQuot }_{C}\right) \cdot c\left(\mathcal{T}_{n}\right)
$$

with the factorization

$$
W=\mathrm{A}^{g-1} \cdot \mathrm{C}_{1}^{c_{1}\left(\beta_{1}\right)} \cdots \mathrm{C}_{\ell}^{c_{1}\left(\beta_{\ell}\right)} .
$$

We will establish that the $x_{m}$-derivatives of the series $\mathrm{A}$ and $\mathrm{C}_{m}$ are rational in $q$ after setting the $x$ 's to 0 . To study these series, we may pick again convenient geometries:

$$
C=\mathbb{P}^{1}, \quad \beta_{i}=\mathcal{O}_{\mathbb{P}^{1}}\left(d_{i}\right)
$$


for arbitrary integers $d_{i}$. Therefore

$$
W=\sum_{n=0}^{\infty} q^{n}(-1)^{n} \cdot \int_{\operatorname{Quot}_{\mathbb{P}^{1}}\left(\mathbb{C}^{N}, n\right)} c_{x_{1}}\left(\mathcal{O}\left(d_{1}\right)^{[n]}\right) \cdots c_{x_{\ell}}\left(\mathcal{O}\left(d_{\ell}\right)^{[n]}\right) \cdot c\left(\operatorname{TQuot}_{\mathbb{P}^{1}}\right) \cdot c\left(\mathcal{T}_{n}\right) .
$$

It suffices to show the rationality of the $x_{m}$-derivatives of $W$.

We use Atiyah-Bott equivariant localization to compute (26). We let the torus $\mathbb{C}^{\star}$ act on $\mathbb{C}^{N}$ with weights

$$
w_{1}, \ldots, w_{N},
$$

thus inducing an action on $\operatorname{Quot}_{\mathbb{P}^{1}}\left(\mathbb{C}^{N}, n\right)$. The fixed loci were noted in OP1 to be isomorphic to

$$
C^{\left[n_{1}\right]} \times \cdots \times C^{\left[n_{N}\right]}=\mathbb{P}^{n_{1}} \times \cdots \times \mathbb{P}^{n_{N}}
$$

for partitions

$$
n_{1}+\ldots+n_{N}=n \text {. }
$$

Equivariant localization applied to (26) thus yields

$$
W=\sum_{n=0}^{\infty} q^{n} \cdot \sum_{n_{1}+\ldots+n_{N}=n} \int_{\mathbb{P}^{n_{1}} \times \cdots \times \mathbb{P}^{n_{N}}} \operatorname{Contr}\left(n_{1}, \ldots, n_{N}\right) .
$$

The expression

$$
\operatorname{Contr}\left(n_{1}, \ldots, n_{N}\right)
$$

encodes the contribution of the fixed loci. In the absence of the descendent classes $c_{x}\left(\mathcal{O}(d)^{[n]}\right)$, the contribution was determined explicitly in OP1] in the proof of Theorem 18 , via a calculation of the normal bundles of the fixed loci. The answer is

$$
\operatorname{Contr}\left(n_{1}, \ldots, n_{N}\right)=(-1)^{n N+\left(\begin{array}{c}
N \\
2
\end{array}\right)} \cdot \Phi_{1}\left(h_{1}\right)^{n_{1}} \ldots \Phi_{N}\left(h_{N}\right)^{n_{N}} \cdot \Psi\left(h_{1}, \ldots, h_{n}\right)
$$

for the rational functions

$$
\begin{gathered}
\Phi_{i}\left(h_{i}\right)=\prod_{j=1}^{N}\left(1-h_{i}+w_{i}-w_{j}\right) \cdot \prod_{j \neq i}\left(h_{i}+w_{j}-w_{i}\right)^{-1}, \\
\Psi=\prod_{i<j}\left(h_{i}-h_{j}+w_{j}-w_{i}\right)^{2} \cdot \prod_{i, j}\left(1+h_{i}+w_{j}-w_{i}\right) \cdot\left(1+h_{i}-h_{j}+w_{j}-w_{i}\right)^{-1} \\
\cdot \prod_{j \neq i}\left(h_{i}+w_{j}-w_{i}\right)^{-1} .
\end{gathered}
$$

We must modify these rational functions to account for the descendent insertions.

We will use Lemma 27 of [OP1]. For $N=1$, over $C^{[n]}=\mathbb{P}^{n}$, the tautological classes can be expressed in $K$-theory as

$$
\mathcal{O}(d)^{[n]}=(d+1) \cdot \mathcal{O}_{\mathbb{P}^{n}}+(-d+n-1) \cdot \mathcal{O}_{\mathbb{P}^{n}}(-1) .
$$


Letting the torus act on $\mathcal{O}(d)^{[n]}$ fiberwise with weight $w$ and writing $h$ for the hyperplane class, we obtain

$$
c_{x}\left(\mathcal{O}(d)^{[n]}[w]\right)=(1+x w)^{d+1} \cdot(1+x(w-h))^{-d+n-1} .
$$

For $N>1$, restricting $\mathcal{O}(d)^{[n]}$ to the fixed locus

$$
\mathbb{P}^{n_{1}} \times \cdots \times \mathbb{P}^{n_{N}}
$$

yields

$$
c_{x}\left(\mathcal{O}(d)^{[n]}\right)=\prod_{i=1}^{N}\left(1+x w_{i}\right)^{d+1} \cdot \prod_{i=1}^{N}\left(1+x\left(w_{i}-h_{i}\right)\right)^{-d+n_{i}-1},
$$

with $h_{i}$ denoting the hyperplane classes of each factor. The new contributions to (27) thus become

$$
\operatorname{Contr}\left(n_{1}, \ldots, n_{N}\right)=(-1)^{n N+\left(\begin{array}{c}
N \\
2
\end{array}\right)} \cdot \Phi_{1}\left(h_{1}\right)^{n_{1}} \cdots \Phi_{N}\left(h_{N}\right)^{n_{N}} \cdot \Psi\left(h_{1}, \ldots, h_{n}\right)
$$

for the new rational functions

$$
\begin{gathered}
\Phi_{i}\left(h_{i}\right)=\prod_{j=1}^{N}\left(1-h_{i}+w_{i}-w_{j}\right) \cdot \prod_{j \neq i}\left(h_{i}+w_{j}-w_{i}\right)^{-1} \cdot \prod_{m=1}^{\ell}\left(1+x_{m}\left(w_{i}-h_{i}\right)\right), \\
\Psi=\prod_{i<j}\left(h_{i}-h_{j}+w_{j}-w_{i}\right)^{2} \cdot \prod_{i, j}\left(1+h_{i}+w_{j}-w_{i}\right) \cdot\left(1+h_{i}-h_{j}+w_{j}-w_{i}\right)^{-1} \\
\cdot \prod_{j \neq i}\left(h_{i}+w_{j}-w_{i}\right)^{-1} \cdot \prod_{i} \prod_{m=1}^{\ell}\left(1+x_{m}\left(w_{i}-h_{i}\right)\right)^{-1-d_{m}} \cdot\left(1+x_{m} w_{i}\right)^{d_{m}+1} .
\end{gathered}
$$

We conclude

$$
\begin{aligned}
& W=\sum_{n=0}^{\infty} q^{n}(-1)^{n N+\left(\begin{array}{c}
N \\
2
\end{array}\right)} \\
& \cdot \sum_{n_{1}+\ldots+n_{N}=n}\left[h_{1}^{n_{1}} \cdots h_{N}^{n_{N}}\right] \Phi_{1}\left(h_{1}\right)^{n_{1}} \cdots \Phi_{N}\left(h_{N}\right)^{n_{N}} \cdot \Psi\left(h_{1}, \ldots, h_{n}\right) .
\end{aligned}
$$

The brackets in the above series are used to denote the coefficient of the relevant monomial.

By Lagrange-Bürmann's formula G], we obtain

$$
W=(-1)^{\left(\begin{array}{c}
N \\
2
\end{array}\right)} \cdot \frac{\Psi}{K}\left(h_{1}, \ldots, h_{N}\right)
$$

where as usual

$$
K=\prod_{i=1}^{N}\left(1-h_{i} \cdot \frac{\Phi_{i}^{\prime}\left(h_{i}\right)}{\Phi_{i}\left(h_{i}\right)}\right),
$$

and $h_{i}$ is the solution to the equation

$$
(-1)^{N} q=\frac{h_{i}}{\Phi_{i}\left(h_{i}\right)}
$$


satisfying $h_{i}(q=0)=0$. At the end, we also set the equivariant weights $w_{i}$ equal to zero.

We define

$$
\mathbf{X}(g)=\prod_{j=1}^{N} \frac{g+w_{j}}{1-g-w_{j}} \cdot \prod_{m=1}^{\ell} \frac{1}{1-x_{m} g}
$$

We then have

$$
\frac{h_{i}}{\Phi_{i}\left(h_{i}\right)}=\mathrm{X}\left(h_{i}-w_{i}\right) .
$$

Let $g_{1}, \ldots, g_{N}$ be the solutions 9 to

$$
\mathbf{X}(g)=(-1)^{N} q
$$

with initial conditions

$$
g_{i}(q=0)=-w_{i} .
$$

The $g_{i}$ are power series in $q$ whose coefficients are rational functions in $\left\{w_{i}\right\}$ and $\left\{x_{m}\right\}$. Thus

$$
h_{i}=g_{i}+w_{i}
$$

is a solution to

$$
\frac{h_{i}}{\Phi_{i}\left(h_{i}\right)}=(-1)^{N} q
$$

with $h_{i}(q=0)=0$.

We can easily check

$$
K\left(h_{1}, \ldots, h_{N}\right)=\prod_{i=1}^{N} h_{i} \frac{d}{d h_{i}} \log \frac{h_{i}}{\Phi_{i}\left(h_{i}\right)}=\prod_{i=1}^{N} h_{i} \frac{d}{d g_{i}} \log \mathbf{X}\left(g_{i}\right) .
$$

Furthermore,

$$
\begin{aligned}
\Psi\left(h_{1}, \ldots, h_{N}\right)= & \prod_{i=1}^{N} h_{i} \cdot \prod_{i<j}\left(g_{i}-g_{j}\right)^{2} \cdot \prod_{i, j}\left(1+g_{i}+w_{j}\right) \cdot\left(1+g_{i}-g_{j}\right)^{-1} \cdot\left(g_{i}+w_{j}\right)^{-1} \\
& \cdot \prod_{i=1}^{N} \prod_{m=1}^{\ell}\left(1-g_{i} x_{m}\right)^{-1-d_{m}} \cdot\left(1+x_{m} w_{i}\right)^{d_{m}+1} .
\end{aligned}
$$

The expressions for $K$ and $\Psi$ are evidently symmetric in $g_{1}, \ldots, g_{N}$, except for the factor $\prod_{i=1}^{N} h_{i}$ which appears in both. Hence the quotient

$$
\frac{\Psi}{K}\left(h_{1}, \ldots, h_{N}\right)
$$

can be expressed as a rational function in $\left\{g_{i}\right\},\left\{w_{i}\right\}$, and $\left\{x_{m}\right\}$ which is symmetric in the $\left\{g_{i}\right\}$.

\footnotetext{
${ }^{9}$ There are other roots which we will deal with later. See equation (29).
} 
We rewrite the equation $\mathrm{X}(g)=(-1)^{N} q$ as $\mathrm{P}(g)=0$, where

$$
\begin{aligned}
\mathrm{P}(g) & =\prod_{i=1}^{N}\left(g+w_{i}\right)-q \prod_{i=1}^{N}\left(g+w_{i}-1\right) \prod_{m=1}^{\ell}\left(1-x_{m} g\right) \\
& =\sum_{j=0}^{N+\ell} \mathrm{P}_{j} g^{j} .
\end{aligned}
$$

The $\left\{g_{i}\right\}$ are roots of $\mathrm{P}$. Hence, $\mathrm{P}$ factors as

$$
\begin{aligned}
\mathrm{P}(g) & =\prod_{i=1}^{N}\left(g-g_{i}\right) \cdot\left(f_{\ell} g^{\ell}+f_{\ell-1} g^{\ell-1}+\cdots+f_{0}\right) \\
& =\left(g^{N}+e_{N-1} g^{N-1}+\cdots+e_{0}\right) \cdot\left(f_{\ell} g^{\ell}+f_{\ell-1} g^{\ell-1}+\cdots+f_{0}\right)
\end{aligned}
$$

where $e_{i}$ is $(-1)^{i}$ times the $(N-i)$ th elementary symmetric function in $g_{i}$, and the $f_{m}$ are power series in $q$ with coefficients given by rational functions of $\left\{w_{i}\right\}$ and $\left\{x_{m}\right\}$. Now, setting $q=0$, we see that

$$
\begin{aligned}
\prod_{i=1}^{N}\left(g+w_{i}\right) & =\mathrm{P}(g, q=0) \\
& =\prod_{i=1}^{N}\left(g-g_{i}(0)\right) \cdot\left(f_{\ell}(0) g^{\ell}+f_{\ell-1}(0) g^{\ell-1}+\cdots+f_{0}(0)\right) \\
& =\prod_{i=1}^{N}\left(g+w_{i}\right) \cdot\left(f_{\ell}(0) g^{\ell}+f_{\ell-1}(0) g^{\ell-1}+\cdots+f_{0}(0)\right) .
\end{aligned}
$$

It follows then that $f_{m}(0)=0$ for $m>0$, and $f_{0}(0)=1$.

We claim that both $\left\{e_{i}\right\}$ and $\left\{f_{m}\right\}$ are series in $q$ whose coefficients are polynomials in $\left\{w_{i}\right\}$ and $\left\{x_{m}\right\}$. We will abbreviate this by saying that they "are polynomial". So far, we can see that this is true up to order 0 . Let us assume, by induction, that this is true to order $p$.

For $m=\ell, \ell-1, \ldots, 0$, we compare the coefficient of $g^{m+N}$ in the expressions (28) and (29). We have

$$
f_{m}+\sum_{k \geq 1} f_{m+k} e_{N-k}=\mathrm{P}_{m+N}
$$

Here, $k$ goes up to the minimum of $N$ and $\ell-m$, and the sum is empty for $m=\ell$. By inducting on $m$, we may assume that all the $f_{m+k}$ are polynomial to order $p+1$. Since these $f_{m+k}$ also have no constant term, the $(p+1)$ st term of $e_{N-k}$ is not needed to compute the $(p+1)$ st term of $f_{m+k} e_{N-k}$. In addition, $\mathrm{P}_{m+N}$ is known exactly and is polynomial. Hence, we see that $f_{m}$ is polynomial to order $p+1$. 
Now, for $i=0, \ldots, N-1$, we compare the coefficients of $g^{i}$ in the expressions (28) and (29). We have

$$
e_{i} f_{0}+\sum_{k \geq 1} e_{i-k} f_{k}=\mathrm{P}_{i}
$$

By inducting on $i$, we may assume that the $e_{i-k}$ are polynomial to order $p+1$. We also know the $f_{k}$ are polynomial to order $p+1$. We know that $f_{0}$ starts with 1 , so it has a multiplicative inverse which is also polynomial to order $p+1$. It follows that $e_{i}$ is polynomial to order $p+1$. Our induction on $p$ is complete.

We would like to see that any order derivative

$$
\left.\frac{\partial^{k_{1}}}{\partial^{k_{1}} x_{1}} \cdots \frac{\partial^{k_{\ell}}}{\partial^{k_{\ell}} x_{\ell}}\right|_{x_{1}=\ldots=x_{\ell}=0}
$$

of $\Psi / K$ is a rational function in $q$, after setting the $w^{\prime} s$ to zero. This will follow from the observations below.

Fix a rational function $\mathrm{R}$ of $\left\{g_{i}\right\},\left\{w_{i}\right\},\left\{x_{m}\right\}$ and $q$, which is symmetric in the $\left\{g_{i}\right\}$. Of course $\mathrm{R}$ can be rewritten as a rational function of $\left\{e_{i}\right\},\left\{w_{i}\right\},\left\{x_{m}\right\}$ and $q$. Setting $\left\{w_{i}=0\right\}$ and $\left\{x_{m}=0\right\}$ in (28) and (29), we obtain

$$
g^{N}-q(g-1)^{N}=\left(g^{N}+\bar{e}_{N-1} g^{N-1}+\cdots+\bar{e}_{0}\right) \cdot\left(\bar{f}_{\ell} g^{\ell}+\bar{f}_{\ell-1} g^{\ell-1}+\cdots+\bar{f}_{0}\right)
$$

(where the bar indicates the evaluation at 0). These substitutions make sense since we have established polynomiality of the coefficients in the previous paragraph. Consequently

$$
\bar{f}_{0}=1-q
$$

and $\bar{f}_{m}=0$ for $m>0$. Then $\bar{e}_{i}$ is the coefficient of $g^{i}$ in

$$
\bar{f}_{0}^{-1}\left(g^{N}-q(g-1)^{N}\right)=\frac{g^{N}-q(g-1)^{N}}{1-q},
$$

which is clearly a rational function in $q$. It follows that $\overline{\mathrm{R}}$ is a rational function in $q$.

Next, we claim that the derivatives

$$
\frac{\partial \mathrm{R}}{\partial x_{j}}
$$

are also given by rational functions in $\left\{g_{i}\right\},\left\{w_{i}\right\},\left\{x_{m}\right\}$ and $q$, symmetric in the $\left\{g_{i}\right\}$ 's. (We are viewing $g_{i}$ as functions of the independent variables $\left\{w_{i}\right\},\left\{x_{m}\right\}$ and $q$. .) Indeed, we have

$$
\frac{\partial \mathrm{R}}{\partial x_{j}}=\sum_{i=1}^{N} \frac{\partial \mathrm{R}}{\partial g_{i}} \cdot \frac{\partial g_{i}}{\partial x_{j}}+\partial_{j} \mathrm{R} .
$$

(Here $\partial_{j} \mathrm{R}$ means to take the $x_{j}$-derivative treating the $\left\{g_{i}\right\}$ as constants.) The second term $\partial_{j} \mathrm{R}$ is manifestly symmetric in the $g$ 's since $\mathrm{R}$ is. Again because $\mathrm{R}$ is symmetric, 
we see that transposing $g_{i}$ and $g_{k}$ turns

$$
\frac{\partial \mathrm{R}}{\partial g_{i}} \text { into } \frac{\partial \mathrm{R}}{\partial g_{k}} \text {. }
$$

For each fixed $i$, the derivative $\frac{\partial g_{i}}{\partial x_{j}}$ can be expressed as a rational function in the $g_{i}$ (but no other $g$ 's), the $\left\{w_{i}\right\},\left\{x_{m}\right\}$, and $q$ by implicit differentiation applied to

$$
\mathrm{X}\left(g_{i}\right)=(-1)^{N} q .
$$

Replacing $g_{i}$ with $g_{k}$ in this formula therefore yields the formula for $\frac{\partial g_{k}}{\partial x_{j}}$. The claim now follows since we sum over all $i$.

Inductively, it follows that all higher derivatives of $\mathrm{R}$ are rational functions of $\left\{g_{i}\right\}$, $\left\{w_{i}\right\},\left\{x_{m}\right\}$ and $q$, symmetric in the $\left\{g_{i}\right\}$. This completes the proof of Theorem 2,

2.3. Example. The proof of the Theorem gives an effective algorithm of computing the descendent series for $\beta=0$. We illustrate the case

$$
N=2, \alpha_{1}=\mathcal{O}_{X}, k_{1}=1, \ell=1 .
$$

Thus

$$
Z=\sum_{n=0}^{\infty} q^{n} \cdot \int_{\left[\operatorname{Quot}_{X}\left(\mathbb{C}^{2}, n\right)\right]^{\operatorname{vir}}} \operatorname{ch}_{1}\left(\mathcal{O}^{[n]}\right) \cdot c\left(T^{\mathrm{vir}} \text { Quot }\right) .
$$

When $N=1$, for the Hilbert scheme of points, the boundary insertion $c_{1}\left(\mathcal{O}^{[n]}\right)$ plays an important role in the formalism of [Le].

Setting

$$
W=\sum_{n=0}^{\infty} q^{n} \cdot \int_{\left[\operatorname{Quot}_{X}\left(\mathbb{C}^{2}, n\right)\right]^{\mathrm{vir}}} c_{x}\left(\mathcal{O}^{[n]}\right) \cdot c\left(T^{\mathrm{vir}} \text { Quot }\right)
$$

we have

$$
W=\mathrm{A}^{K_{X}^{2}}
$$

for some universal series A. No other universal functions are needed in this case. Thus

$$
Z=\left.\frac{\partial W}{\partial x}\right|_{x=0}=\left.K_{X}^{2} \cdot \mathrm{A}^{K_{X}^{2}-1} \cdot \frac{\partial \mathrm{A}}{\partial x}\right|_{x=0} .
$$

We already calculated

$$
\left.\mathrm{A}\right|_{x=0}=\frac{(1-q)^{2} \cdot\left(1-6 q+q^{2}\right)}{(1-4 q)^{2}}
$$

in Theorem 18 of [OP1]. We furthermore claim

$$
\left.\frac{\partial \mathrm{A}}{\partial x}\right|_{x=0}=\frac{2 q^{2} \cdot\left(1-12 q-33 q^{2}+8 q^{3}\right)}{(1-4 q)^{3}} .
$$

This follows by the proof of Theorem 2. Indeed, we have

$$
\mathrm{A}^{-1}=\sum_{q=0}^{\infty}(-q)^{n} \int_{\operatorname{Quot}_{\mathbb{P}}\left(\mathbb{C}^{2}, n\right)} c_{x}\left(\mathcal{O}^{[n]}\right) \cdot c\left(T \operatorname{Quot}_{\mathbb{P}^{1}}\right) \cdot c\left(\mathcal{T}_{n}\right)=-\frac{\Psi}{K}\left(g_{1}+w_{1}, g_{2}+w_{2}\right)
$$


where $g_{1}, g_{2}$ solve the equation

$$
\left(g+w_{1}\right) \cdot\left(g+w_{2}\right)=q \cdot\left(1-g-w_{1}\right) \cdot\left(1-g-w_{2}\right) \cdot(1-x g) .
$$

The expressions for $\Psi, \Phi_{i}, K$ are explicitly given in the proof of the Theorem. Substituting and carrying out the implicit differentiation with respect to $x$, we arrive at expression (30) claimed above.

\section{Descendent series for the Hilbert scheme: Theorem 3}

3.1. Descendents. The argument of Theorem 1 extends to prove the more general descendent claim of Theorem 3 . For each $K$-theory class $\alpha$ on $X$, we have defined

$$
\alpha^{[n]}=\mathbf{R} \pi_{\star}\left(\mathcal{Q} \otimes p^{\star} \alpha\right)
$$

on $\operatorname{Quot}_{X}\left(\mathbb{C}^{N}, \beta, n\right)$. The descendent series is given by

$$
\begin{aligned}
& Z_{X, N, \beta}\left(\alpha_{1}, \ldots, \alpha_{\ell} \mid k_{1}, \ldots, k_{\ell}\right)= \\
& \sum_{n \in \mathbb{Z}} q^{n} \cdot \int_{\left[\operatorname{Quot}_{X}\left(\mathbb{C}^{N}, \beta, n\right)\right]^{\mathrm{vir}}} \operatorname{ch}_{k_{1}}\left(\alpha_{1}^{[n]}\right) \cdots \operatorname{ch}_{k_{\ell}}\left(\alpha_{\ell}^{[n]}\right) \cdot c\left(T^{\mathrm{vir}} \text { Quot }\right) .
\end{aligned}
$$

To establish Theorem 3 , we set $N=1$, and show that the series

$$
Z_{X, 1, \beta}\left(\alpha_{1}, \ldots, \alpha_{\ell} \mid k_{1}, \ldots, k_{\ell}\right) \in \mathbb{Q}((q))
$$

is the Laurent expansion of a rational function for a nonsingular projective simply connected surface $X$.

\subsection{Proof of Theorem 3 ,}

3.2.1. Hilbert schemes of points. We use again the isomorphism

$$
\operatorname{Quot}_{X}\left(\mathbb{C}^{1}, \beta, n\right) \simeq X^{[m]} \times \mathbb{P}, \quad m=n+\frac{\beta\left(\beta+K_{X}\right)}{2}
$$

where $\mathbb{P}$ denotes the linear system $|\beta|$. We will study the series

$$
Z=\sum_{n \in \mathbb{Z}} q^{n} \cdot \int_{X^{[m]} \times \mathbb{P}} \operatorname{ch}_{k_{1}}\left(\alpha_{1}^{[n]}\right) \cdots \mathrm{ch}_{k_{\ell}}\left(\alpha_{\ell}^{[n]}\right) \cdot \mathrm{e}(\mathrm{Obs}) \cdot \frac{c\left(T X^{[m]}\right) c(T \mathbb{P})}{c(\mathrm{Obs})} .
$$

We identify the tautological structures appearing in (31). The universal quotient over Quot $_{X}\left(\mathbb{C}^{1}, \beta, n\right) \times X$ can be expressed in $K$-theory as

$$
\mathcal{Q}=\mathcal{O}-\mathcal{I}_{\mathcal{W}} \otimes \mathcal{O}(-\beta) \otimes \mathcal{L}^{-1}=\mathcal{O}-\mathcal{O}(-\beta) \otimes \mathcal{L}^{-1}+\mathcal{O}_{\mathcal{W}} \otimes \mathcal{O}(-\beta) \otimes \mathcal{L}^{-1}
$$

where $\mathcal{W}$ denotes the universal subscheme of $X^{[m]} \times X$, and

$$
\mathcal{L}=\mathcal{O}_{\mathbb{P}}(1) \rightarrow \mathbb{P}
$$

denotes the tautological bundle. As a result

$$
\alpha_{\text {Quot }}^{[n]}=H^{\bullet}(\alpha) \otimes \mathcal{O}-H^{\bullet}(\widetilde{\alpha}) \otimes \mathcal{L}^{-1}+\widetilde{\alpha}_{\text {Hilb }}^{[m]} \otimes \mathcal{L}^{-1}
$$


where $\widetilde{\alpha}=\alpha \otimes \mathcal{O}(-\beta)$. We have indicated by subscripts the locations of the tautological constructions. Let $\zeta=c_{1}(\mathcal{L})$. Thus

$$
\operatorname{ch}\left(\alpha_{\text {Quot }}^{[n]}\right)=\chi(\alpha)-\chi(\widetilde{\alpha}) \cdot e^{-\zeta}+\operatorname{ch}\left(\widetilde{\alpha}_{\text {Hilb }}^{[m]}\right) \cdot e^{-\zeta}
$$

which, in fixed degree $k>0$, becomes

$$
\operatorname{ch}_{k}\left(\alpha_{\text {Quot }}^{[n]}\right)=-\chi(\widetilde{\alpha}) \cdot \frac{(-\zeta)^{k}}{k !}+\sum_{j=0}^{k} \operatorname{ch}_{j}\left(\widetilde{\alpha}_{\text {Hilb }}^{[m]}\right) \cdot \frac{(-\zeta)^{k-j}}{(k-j) !} .
$$

After multiplying out the different Chern characters appearing in (31), we are led to expressions of the form

$$
\sum_{m=0}^{\infty} q^{m} \cdot \int_{X^{[m]} \times \mathbb{P}} \zeta^{k} \cdot \operatorname{ch}_{k_{1}}\left(\alpha_{1}^{[m]}\right) \cdots \mathrm{ch}_{k_{\ell}}\left(\alpha_{\ell}^{[m]}\right) \cdot \mathrm{e}(\mathrm{Obs}) \cdot \frac{c\left(T X^{[m]}\right) c(T \mathbb{P})}{c(\mathrm{Obs})} .
$$

Here, we have changed notation by removing the tilde's from the $\alpha$ 's and relabeling indices 10 Unless specified otherwise, all tautological structures $\alpha^{[m]}$ are from now on understood to be over the Hilbert scheme of points $X^{[m]}$.

We will consider two cases depending upon the geometric genus of the simply connected surface $X$. Furthermore, when the genus is positive, we first discuss surfaces which are minimal, and then consider their blowups.

3.2.2. Minimal surfaces with $p_{g}>0$. Assume that $X$ is simply connected minimal surface. Then $X$ is either a $K 3$ surface, an elliptic surface, or a surface of general type.

- For $K 3$ surfaces, the virtual fundamental class vanishes due to the presence of a trivial factor in the obstruction bundle, unless $\beta=m=0$ [MOP1]. There is nothing to prove in the $K 3$ case.

- If $X$ minimal of general type, the virtual fundamental class of $\operatorname{Quot}_{X}\left(\mathbb{C}^{1}, n, \beta\right)$ was shown to vanish in OP1, Section 5.3.3], unless

(i) $\beta=0$ or

(ii) $\beta=K_{X}$ and $m=0$.

There is nothing to prove in case (ii). When $\beta=0$, we can use Theorem 2 or alternatively, we can argue as follows. We have

$$
\text { Obs }=\left(\left(K_{X}\right)^{[m]}\right)^{\vee},
$$

see for instance (6). The series (32) becomes

$$
Z=\sum_{m=0}^{\infty} q^{m} \int_{X^{[m]}} \operatorname{ch}_{k_{1}}\left(\alpha_{1}^{[m]}\right) \cdots \operatorname{ch}_{k_{\ell}}\left(\alpha_{\ell}^{[m]}\right) \cdot \mathrm{e}\left(\left(K_{X}^{[m]}\right)^{\vee}\right) \cdot \frac{c\left(T X^{[m]}\right)}{c\left(\left(K_{X}^{[m]}\right)^{\vee}\right)}
$$

\footnotetext{
${ }^{10}$ The overall $q$ shift does not affect rationality.
} 
We conclude by Proposition 3 below.

- Let $X \rightarrow C$ be a minimal elliptic surface with $p_{g}>0$. Since $X$ is simply connected, we must have $C=\mathbb{P}^{1}$, by [Fr, Lemma VII.14]. We first argue that $\beta$ must be a multiple of the fiber. Note that $\beta$ must be effective for the Quot scheme to be nonempty. Furthermore, the expression for the obstruction bundle (6),

$$
\text { Obs }=\left(H^{1}(M)-H^{0}(M)\right) \otimes \mathcal{L}+\left(M^{[m]}\right)^{\vee} \otimes \mathcal{L}+\mathbb{C}^{p_{g}},
$$

shows that the virtual fundamental class vanishes if

$$
H^{0}(M)=0 \Longleftrightarrow H^{0}\left(K_{X}-\beta\right)=0
$$

due to the presence of the trivial factor. We may therefore assume $K_{X}-\beta$ is effective. Since $X$ is minimal, we find

$$
K_{X}=\left(p_{g}-1\right) f
$$

Since

$$
\left(p_{g}-1\right) f=\beta+\left(K_{X}-\beta\right)
$$

is an effective decomposition, $\beta$ must be supported on fibers. By Zariski's Lemma, $\beta^{2} \leq 0$ and $\beta \cdot K_{X}=0$. If $\beta^{2}<0$ then

$$
\beta \cdot\left(\beta-K_{X}\right)<0
$$

When inequality (33) is satisfied, the proof of Proposition 22 of [OP1] shows that the virtual fundamental class vanishes. Proposition 22 of [OP1 is stated for surfaces of general type, but the same argument applies here as well 11 Thus

$$
\beta^{2}=0
$$

so by Zariski's Lemma $\beta=$ af for $0 \leq a \leq p_{g}-1$.

Recording that

$$
\chi(\mathcal{O}(a f))=1+p_{g}, \quad h^{0}(\mathcal{O}(a f))=a+1,
$$

we find that (6) becomes

$$
\text { Obs }=-\mathbb{C}^{p_{g}-a} \otimes \mathcal{L}+\left(M^{[m]}\right)^{\vee} \otimes \mathcal{L}+\mathbb{C}^{p_{g}}
$$

over $X^{[m]} \times \mathbb{P}^{a}$. We then obtain

$$
\begin{aligned}
\mathrm{e}(\text { Obs }) & =\left[c(\mathcal{L})^{a-p_{g}} \cdot c\left(\left(M^{[m]}\right)^{\vee} \otimes \mathcal{L}\right)\right]_{(a+m)} \\
& =\left[(1+\zeta)^{a-p_{g}} \cdot \sum_{k=0}^{m}(1+\zeta)^{k} c_{m-k}\left(\left(M^{[m]}\right)^{\vee}\right)\right]_{(a+m)} .
\end{aligned}
$$

\footnotetext{
${ }^{11}$ This can also be seen via (5) since $\mathrm{Hilb}_{\beta}$ has negative virtual dimension.
} 
The exponents of the hyperplane class $\zeta$ over $\mathbb{P}^{a}$ must be bounded by $a$. Thus, for degree reasons, the only contribution reaching the necessary degree $a+m$ occurs for $k=0$ and in this case

$$
\mathrm{e}(\mathrm{Obs})=\left[(1+\zeta)^{a-p_{g}}\right]_{(a)} \times c_{m}\left(\left(M^{[m]}\right)^{\vee}\right)=\left(\begin{array}{c}
a-p_{g} \\
a
\end{array}\right)[\mathrm{pt}] \times \mathrm{e}\left(\left(M^{[m]}\right)^{\vee}\right)
$$

over $\mathbb{P}^{a} \times X^{[m]}$.

As a result of the above calculation, the series (32),

$$
\sum_{m=0}^{\infty} q^{m} \int_{X^{[m]} \times \mathbb{P} a} \zeta^{k} \cdot \operatorname{ch}_{k_{1}}\left(\alpha_{1}^{[m]}\right) \cdots \operatorname{ch}_{k_{\ell}}\left(\alpha_{\ell}^{[m]}\right) \cdot \mathrm{e}(\mathrm{Obs}) \cdot \frac{c\left(T X^{[m]}\right) c(T \mathbb{P})}{c(\mathrm{Obs})},
$$

vanishes for $k>0$. For $k=0$, the expression simplifies to

$$
\left(\begin{array}{c}
a-p_{g} \\
a
\end{array}\right) \cdot \sum_{m=0}^{\infty} q^{m} \int_{X^{[m]}} \operatorname{ch}_{k_{1}}\left(\alpha_{1}^{[m]}\right) \cdots \operatorname{ch}_{k_{\ell}}\left(\alpha_{\ell}^{[m]}\right) \cdot \mathrm{e}\left(\left(M^{[m]}\right)^{\vee}\right) \cdot \frac{c\left(T X^{[m]}\right)}{c\left(\left(M^{[m]}\right)^{\vee}\right)} .
$$

Proposition 3 below completes the argument.

Theorem 3 is established for all simply connected minimal surfaces with $p_{g}>0$.

3.2.3. Further descendent rationality. We prove here the following result that was used in Subsection 3.2.2.

Proposition 3. The generating series

$$
\sum_{n=0}^{\infty} q^{n} \cdot \int_{X^{[n]}} \operatorname{ch}_{k_{1}}\left(\alpha_{1}^{[n]}\right) \cdots \operatorname{ch}_{k_{\ell}}\left(\alpha_{\ell}^{[n]}\right) \cdot \mathrm{e}\left(\left(M^{[n]}\right)^{\vee}\right) \cdot \frac{c\left(T X^{[n]}\right)}{c\left(\left(M^{[n]}\right)^{\vee}\right)}
$$

is a rational function in $q$ for all pairs $(X, M)$.

Proof. The proof is similar to that of Theorem 2, using the methods developed in [OP1]. Just as in Theorem 2, we may assume that

$$
\text { rank } \alpha_{i}=1 \text { for all } 1 \leq i \leq \ell .
$$

Expressing the Chern character in terms of Chern classes, it suffices to show that the series

$$
Z=\sum_{n=0}^{\infty} q^{n} \int_{X^{[n]}} c_{k_{1}}\left(\alpha_{1}^{[n]}\right) \ldots c_{k_{\ell}}\left(\alpha_{\ell}^{[n]}\right) \cdot \mathrm{e}\left(\left(M^{[n]}\right)^{\vee}\right) \cdot \frac{c\left(T X^{[n]}\right)}{c\left(\left(M^{[n]}\right)^{\vee}\right)}
$$

is a rational function in $q$.

Let $x_{1}, \ldots, x_{\ell}$ be formal variables. Write

$$
c_{x}=1+x c_{1}+x^{2} c_{2}+\ldots
$$

for the total Chern class, and set

$$
W=\sum_{n=0}^{\infty} q^{n} \int_{X^{[n]}} c_{x_{1}}\left(\alpha_{1}^{[n]}\right) \ldots c_{x_{\ell}}\left(\alpha_{\ell}^{[n]}\right) \cdot \mathrm{e}\left(\left(M^{[n]}\right)^{\vee}\right) \cdot \frac{c\left(T X^{[n]}\right)}{c\left(\left(M^{[n]}\right)^{\vee}\right)} .
$$


The series $Z$ is found by extracting the coefficient of $x_{1}^{k_{1}} \cdots x_{\ell}^{k_{\ell}}$ in $W$ :

$$
Z=\left.\frac{1}{k_{1} !} \cdots \frac{1}{k_{\ell} !} \cdot \frac{\partial^{k_{1}}}{\partial^{k_{1}} x_{1}} \cdots \frac{\partial^{k_{\ell}}}{\partial^{k_{\ell}} x_{\ell}} W\right|_{x_{1}=\ldots=x_{\ell}=0} .
$$

Now, invoking the universality and multiplicativity results of [EGL, we find the factorization

$$
W=\mathrm{A}^{K_{X}^{2}} \cdot \mathrm{B}^{\chi(X)} \cdot \mathrm{C}^{M^{2}} \cdot \mathrm{D}^{M \cdot K_{X}} \cdot \prod_{i=1}^{\ell} \mathrm{E}_{i}^{c_{1}\left(\alpha_{i}\right) \cdot K_{X}} \cdot \mathrm{F}_{i}^{c_{2}\left(\alpha_{i}\right)} \cdot \mathrm{G}_{i}^{c_{1}\left(\alpha_{i}\right) \cdot M} \cdot \prod_{1 \leq i \leq j \leq \ell} \mathrm{H}_{i j}^{c_{1}\left(\alpha_{i}\right) \cdot c_{1}\left(\alpha_{j}\right)}
$$

in terms of universal series that depend on $q$ and $x_{i}$. To find these series, we can pick convenient geometries. We may assume $M$ is sufficiently positive, so that there exists $C$ a nonsingular connected curve in the linear system $|M|$. As explained in [OP1], we have

$$
\mathrm{e}\left(\left(M^{[n]}\right)^{\vee}\right) \cap\left[X^{[n]}\right]=(-1)^{n} j_{\star}\left[C^{[n]}\right]
$$

where

$$
j: C^{[n]} \hookrightarrow X^{[n]}
$$

is the natural inclusion. By equation (33) of [OP1, we furthermore have

$$
j^{\star} c\left(T X^{[n]}\right)=c\left(\left(K_{C}^{[n]}\right)^{\vee}\right) \cdot c\left(M^{[n]}\right) .
$$

Thus

$$
W=\sum_{n=0}^{\infty}(-q)^{n} \int_{C^{[n]}} c_{x_{1}}\left(\alpha_{1}^{[n]}\right) \cdots c_{x_{\ell}}\left(\alpha_{\ell}^{[n]}\right) \cdot \frac{c\left(\left(K_{C}^{[n]}\right)^{\vee}\right) \cdot c\left(M^{[n]}\right)}{c\left(\left(M^{[n]}\right)^{\vee}\right)}
$$

or equivalently, in terms of Segre classes

$$
\begin{aligned}
\sum_{n=0}^{\infty}(-q)^{n} \cdot \int_{C^{[n]}} s_{x_{1}}\left(\left(-\alpha_{1}\right)^{[n]}\right) \cdots s_{x_{\ell}} & \left(\left(-\alpha_{\ell}\right)^{[n]}\right) \\
& \cdot s_{1}\left((-M)^{[n]}\right) \cdot s_{-1}\left(\left(-K_{C}\right)^{[n]}\right) \cdot s_{-1}\left(M^{[n]}\right) .
\end{aligned}
$$

Here, $\alpha_{1}, \cdots, \alpha_{\ell}, M$ are understood to be restricted from the surface $X$ to the curve $C$.

Using Theorem 3 of [OP1, the last expression can be evaluated in closed form. Under the change of variables

$$
-q=\frac{t}{(1-t)\left(1-x_{1} t\right) \cdots\left(1-x_{\ell} t\right)},
$$

we have

$$
W\left(q, x_{1}, \ldots, x_{\ell}\right)=\prod_{i=1}^{\ell}\left(1-x_{i} t\right)^{-c_{1}\left(\alpha_{i}\right) \cdot M} \cdot(1-t)^{-M^{2}} \cdot(1+t)^{-M \cdot K_{X}} \cdot \mathrm{a}^{K_{X}^{2}}
$$


with 12

It follows that

$$
\mathrm{a}=-\left(\frac{q}{t}\right)^{-2} \cdot \frac{d q}{d t}
$$

$$
\mathrm{B}=1, \quad \mathrm{E}_{\mathrm{i}}=1, \quad \mathrm{~F}_{\mathrm{i}}=1, \quad \mathrm{H}_{i j}=1 .
$$

Furthermore, by universality, expression (35) for $W$ holds for all geometries $\left(X, M, \alpha_{1}, \ldots, \alpha_{\ell}\right)$, not only for those for which $M$ is sufficiently positive.

Identity (35) for the values $x_{1}=\ldots=x_{\ell}=0$ implies

$$
W(q, 0, \ldots, 0)=(1-t)^{-M^{2}} \cdot(1+t)^{-M \cdot K_{X}}
$$

with

$$
q=-\frac{t}{1-t} \Longleftrightarrow t=-\frac{q}{1-q} .
$$

Evidently $W(q, 0, \ldots, 0)$ is a rational function of $t$ and hence also of $q$. In fact, the expression we have obtained,

$$
W(q, 0, \ldots, 0)=(1-q)^{M^{2}} \cdot\left(\frac{1-q}{1-2 q}\right)^{M \cdot K_{X}},
$$

is Corollary 38 of [P1].

However, we can now also go further. We address all derivatives of $W$ with respect to $x_{i}$ via (35), as needed to complete the proof of Proposition 3. Clearly, the derivatives

$$
\left.\frac{\partial^{k_{1}}}{\partial^{k_{1}} x_{1}} \cdots \frac{\partial^{k_{\ell}}}{\partial^{k_{\ell}} x_{\ell}}\right|_{x_{1}=\ldots=x_{\ell}=0} \text { RHS of (35) }
$$

are rational functions in $t$. For the left hand side of (35), we apply the chain rule repeatedly using that

$$
\left.\frac{\partial q}{\partial x_{i}}\right|_{x_{1}=\ldots=x_{\ell}=0}=-\frac{t^{2}}{1-t},,\left.q\right|_{x_{1}=\ldots=x_{\ell}=0}=-\frac{t}{1-t} .
$$

For instance, the $x_{1}$ derivative equals

$$
\left.\frac{d}{d x_{1}} W\left(q, x_{1}, \ldots, x_{\ell}\right)\right|_{x_{1}=\ldots=x_{\ell}=0}=\left.\frac{\partial W}{\partial q}\right|_{\substack{q=-\frac{t}{1-t} \\ x_{1}=\ldots=x_{\ell}=0}} \cdot \frac{-t^{2}}{1-t}+\left.\frac{\partial W}{\partial x_{1}}\right|_{\substack{q=-\frac{t}{1-t} \\ x_{1}=\ldots=x_{\ell}=0}} .
$$

We argued the left hand side is rational in $t$. Since $\frac{\partial W}{\partial q}(q, 0, \ldots, 0)$ is rational in $t$ by (36), we conclude that the same is true about the last term

$$
\left.\frac{\partial W}{\partial x_{1}}\right|_{\substack{q=-\frac{t}{1-t} \\ x_{1}=\ldots=x_{\ell}=0}} .
$$

\footnotetext{
${ }^{12}$ We can show

$$
\mathrm{a}=1-e_{2} t^{2}+2 e_{3} t^{3}-3 e_{4} t^{4}+\ldots
$$
}

where $e_{i}$ are the elementary symmetric functions in $1, x_{1}, \ldots, x_{\ell}$. We do not explain the latter formula for a since it will not be used here. 
Equivalently,

$$
\left.\frac{\partial W}{\partial x_{1}}\right|_{x_{1}=\ldots=x_{\ell}=0}
$$

is rational in $q$. Rationality of the higher order derivatives follows inductively. The proof of Proposition 3 is complete.

3.2.4. Example. We illustrate Proposition 3 with the computation of the series 13

$$
Z=\sum_{n=0}^{\infty} q^{n} \cdot \int_{\left[X^{[n]}\right]^{\mathrm{vir}}} \mathrm{ch}_{1}\left(\mathcal{O}^{[n]}\right) \cdot c\left(T^{\mathrm{vir}} X^{[n]}\right) .
$$

This expression is of the form considered in the Proposition. Indeed, by equation (33) of OP1 we have

$$
\left[X^{[n]}\right]^{\mathrm{vir}}=\mathrm{e}\left(\left(M^{[n]}\right)^{\vee}\right), \quad T^{\mathrm{vir}} X^{[n]}=T X^{[n]}-\left(M^{[n]}\right)^{\vee}
$$

for $M=K_{X}$. Write

$$
W=\sum_{n=0}^{\infty} q^{n} \cdot \int_{\left[X^{[n]}\right]^{\mathrm{vir}}} c_{x}\left(\mathcal{O}^{[n]}\right) \cdot c\left(T^{\mathrm{vir}} X^{[n]}\right) \Longrightarrow Z=\left.\frac{\partial W}{\partial x}\right|_{x=0} .
$$

Equation (35) for $M=K_{X}$ expresses the answer in terms of a single universal series

$$
W=\mathrm{A}^{K_{X}^{2}}
$$

where

$$
\mathrm{A}(q, x)=\frac{1-t^{2} x}{1-t^{2}}, \text { for } q=-\frac{t}{(1-t)(1-t x)} .
$$

Thus

$$
Z=\left.K_{X}^{2} \cdot \mathrm{A}^{K_{X}^{2}-1} \cdot \frac{\partial \mathrm{A}}{\partial x}\right|_{x=0}
$$

By direct computation

$$
\left.\mathrm{A}\right|_{x=0}=\frac{(1-q)^{2}}{1-2 q},\left.\quad \frac{\partial \mathrm{A}}{\partial x}\right|_{x=0}=-\frac{q^{2} \cdot(1-4 q)}{(1-2 q)^{2}} .
$$

In fact, it can easily be shown that all derivatives take the form

$$
\left.\frac{\partial^{k} \mathrm{~A}}{\partial x^{k}}\right|_{x=0}=\frac{\mathrm{P}_{k}(q)}{(1-q)^{2 k-2} \cdot(1-2 q)^{k+1}}
$$

for some polynomials $\mathrm{P}_{k}$. The denominators $1-q$ and $1-2 q$ are consistent with the proof of Theorem 1 .

\footnotetext{
${ }^{13}$ The same calculation can also be carried out using Theorem 2
} 
3.2.5. Non-minimal surfaces with $p_{g}>0$. For non-minimal surfaces, we prove Theorem 3 using the calculations of Section 3.2.2, combined with an observation that we learned from Woonam Lim. Specifically, in the next paragraph, we will explain a special case of Lemma 2 of $[\mathrm{L}$ in our simpler setting. The argument rests on a deeper connection with Seiberg-Witten theory and the notion of simple type.

Let $\widetilde{X}$ denote the blowup of a nonsingular projective simply connected surface $X$ with exceptional divisor $E$. For each curve class $\beta$ on $X$ and each integer $k$, consider the class

$$
\widetilde{\beta}=\beta+k E
$$

on $\tilde{X}$. We assume

$$
\left[\operatorname{Quot}_{X}\left(\mathbb{C}^{1}, \beta, n\right)\right]^{\text {vir }} \neq 0 \text { for some } n \Longrightarrow \beta \cdot\left(\beta-K_{X}\right)=0 .
$$

Assumption (37) is satisfied for all three classes of minimal surfaces considered in Section 3.2 .2 as the reader can immediately verify. We claim that the same holds true on $\widetilde{X}$ :

$$
\left[\operatorname{Quot}_{\widetilde{X}}\left(\mathbb{C}^{1}, \widetilde{\beta}, n\right)\right]^{\text {vir }} \neq 0 \text { for some } n \Longrightarrow \widetilde{\beta} \cdot\left(\widetilde{\beta}-K_{\widetilde{X}}\right)=0 \text {. }
$$

By direct calculation,

$$
\widetilde{\beta} \cdot\left(\widetilde{\beta}-K_{\widetilde{X}}\right)=\beta \cdot\left(\beta-K_{X}\right)-k(k-1)=-k(k-1) \leq 0 .
$$

If the inequality is strict

$$
\widetilde{\beta} \cdot\left(\widetilde{\beta}-K_{\widetilde{X}}\right)<0,
$$

the virtual fundamental class vanishes by the proof of Proposition 22 of [OP1] (as already used in equation (33) $)$. We must therefore have $\widetilde{\beta} \cdot\left(\widetilde{\beta}-K_{\widetilde{X}}\right)=0$.

Applying the argument inductively to a sequence of blowups, we see that if $X$ is a possibly non-minimal surface with $p_{g}>0$, non-zero invariants only arise if

$$
\beta \cdot\left(\beta-K_{X}\right)=0 .
$$

The latter condition can be used to explicitly calculate the virtual fundamental class. Indeed, thanks to (38), and recalling the obstruction bundle from equation (6), we have

$$
\operatorname{rank} \text { Obs }=m+h^{0}(\beta)-1 .
$$

We now use the same reasoning that led to (34). For the current numerics, we similarly compute over $X^{[m]} \times \mathbb{P}$ :

$$
\begin{aligned}
\mathrm{e}(\mathrm{Obs}) & =\left[c(\mathcal{L})^{h^{1}(\beta)-h^{2}(\beta)} \cdot c\left(\left(M^{[m]}\right)^{\vee} \otimes \mathcal{L}\right)\right]_{m+h^{0}(\beta)-1} \\
& =\left[(1+\zeta)^{h^{1}(\beta)-h^{2}(\beta)} \cdot \sum_{k=0}^{m}(1+\zeta)^{k} c_{m-k}\left(\left(M^{[m]}\right)^{\vee}\right)\right]_{m+h^{0}(\beta)-1} \\
& =\left(\begin{array}{c}
h^{1}(\beta)-h^{2}(\beta) \\
h^{0}(\beta)-1
\end{array}\right) \cdot \mathrm{e}\left(\left(M^{[m]}\right)^{\vee}\right) \times[\mathrm{pt}]
\end{aligned}
$$


The argument then is completed in the same fashion as for elliptic surfaces in Section 3.2 .2 by invoking Proposition 3 .

3.2.6. Surfaces with $p_{g}=0$. We establish Theorem 3 for surfaces with $p_{g}=0$. We follow here the proof in Section 1.2 closely. We have

$$
\text { Obs }=H^{1}(M)^{\vee} \otimes \mathcal{L}+\left(M^{[n]}\right)^{\vee} \otimes \mathcal{L} .
$$

By (32), we examine expressions of the form

$$
\sum_{n=0}^{\infty} q^{n} \int_{X^{[n]} \times \mathbb{P}} \zeta^{k+h^{1}(\beta)} \cdot \operatorname{ch}_{k_{1}}\left(\alpha_{1}^{[n]}\right) \cdots \operatorname{ch}_{k_{\ell}}\left(\alpha_{\ell}^{[n]}\right) \cdot \mathrm{e}\left(\mathcal{L} \otimes\left(M^{[n]}\right)^{\vee}\right) \cdot \frac{c\left(T X^{[n]}\right) \cdot c(\mathcal{L})^{\chi(\beta)}}{c\left(\mathcal{L} \otimes\left(M^{[n]}\right)^{\vee}\right)} .
$$

Expanding the terms that involve $\mathcal{L}$ into powers of $\zeta=c_{1}(\mathcal{L})$ as in Proposition 2, we obtain

$$
\begin{aligned}
& \sum_{n=0}^{\infty} q^{n} \int_{X^{[n]} \times \mathbb{P}} \zeta^{k+h^{1}(\beta)} \cdot \operatorname{ch}_{k_{1}}\left(\alpha_{1}^{[n]}\right) \cdots \operatorname{ch}_{k_{\ell}}\left(\alpha_{\ell}^{[n]}\right) \cdot \frac{c\left(T X^{[n]}\right)}{c\left(\left(M^{[n]}\right)^{\vee}\right)} \\
& \cdot\left(\sum_{a=0}^{n} \zeta^{a} \cdot c_{n-a}\left(\left(M^{[n]}\right)^{\vee}\right)\right) \cdot\left(\sum_{j=0}^{\infty}(-1)^{j} \zeta^{j} H_{j}\right) \cdot(1+\zeta)^{\chi(\beta)} .
\end{aligned}
$$

Integrating out the powers of $\zeta$, we equivalently prove the rationality of

$$
\sum_{n=0}^{\infty} q^{n} \int_{X^{[n]}} \operatorname{ch}_{k_{1}}\left(\alpha_{1}^{[n]}\right) \cdots \operatorname{ch}_{k_{\ell}}\left(\alpha_{\ell}^{[n]}\right) \cdot c_{n-a}\left(\left(M^{[n]}\right)^{\vee}\right) \cdot c\left(T X^{[n]}\right) \cdot \frac{P\left(\left(M^{[n]}\right)^{\vee}, B\right)}{c\left(\left(M^{[n]}\right)^{\vee}\right)}
$$

for fixed tuples $\left(a, B, k_{1}, \ldots, k_{\ell}, \alpha_{1}, \ldots, \alpha_{\ell}\right)$. Following the proof of Proposition 2 , we will establish first universality and then rationality for sufficiently positive geometries.

For universality, we first turn all Chern characters into universal expressions in the Chern classes:

$$
\sum_{n=0}^{\infty} q^{n} \int_{X^{[n]}} c_{k_{1}}\left(\alpha_{1}^{[n]}\right) \cdots c_{k_{\ell}}\left(\alpha_{\ell}^{[n]}\right) \cdot c_{n-a}\left(\left(M^{[n]}\right)^{\vee}\right) \cdot c\left(T X^{[n]}\right) \cdot \frac{P\left(\left(M^{[n]}\right)^{\vee}, B\right)}{c\left(\left(M^{[n]}\right)^{\vee}\right)} .
$$

We introduce formal variables $x_{1}, \ldots, x_{\ell}$, and form the generating series

$$
\begin{array}{r}
Y_{X, M}^{(p)}=\sum_{B=\left(b_{1}, \ldots, b_{p}\right)} \frac{z_{1}^{b_{1}}}{b_{1} !} \cdots \frac{z_{p}^{b_{p}}}{b_{p} !} \sum_{n \geq 0} \sum_{a \geq 0} q^{n} t^{a} \cdot \int_{X^{[n]}} c_{x_{1}}\left(\alpha_{1}^{[n]}\right) \cdots c_{x_{\ell}}\left(\alpha_{\ell}^{[n]}\right) c_{n-a}\left(\left(M^{[n]}\right)^{\vee}\right) \\
\cdot c\left(T X^{[n]}\right) \cdot \frac{P\left(\left(M^{[n]}\right)^{\vee}, B\right)}{c\left(\left(M^{[n]}\right)^{\vee}\right)} .
\end{array}
$$


The length of $B$ equals the superscript $p$ appearing on the left hand side. We must extract

$$
\text { Coefficient of } x_{1}^{k_{1}} \cdots x_{\ell}^{k_{\ell}} \cdot \frac{z_{1}^{b_{1}}}{b_{1} !} \cdots \frac{z_{p}^{b_{p}}}{b_{p} !} \cdot t^{a} \text { in } Y_{X, M}^{(p)} .
$$

As in Section 1.3.2, $Y_{X, M}^{(p)}$ is multiplicative and can be factored in terms of several universal power series. It suffices therefore to establish rationality (of the correct coefficient) for special geometries.

Returning to expression (39), we pick a sufficiently positive $M$, and represent $c_{n-a}\left(M^{[n]}\right)$ by the relative Hilbert scheme

$$
(\mathcal{C} / \mathcal{B})^{[n]} \rightarrow \mathcal{B}
$$

of a linear system $|V| \subset|M|$ as in Section 1.3.3. By the arguments of the same Section, it suffices to consider expressions of the form

$\sum_{n=0}^{\infty} q^{n} \int_{(\mathcal{C} / \mathcal{B})^{[n]}} \operatorname{ch}_{k_{1}}\left(j^{\star} \alpha_{1}^{[n]}\right) \cdots \operatorname{ch}_{k_{\ell}}\left(j^{\star} \alpha_{\ell}^{[n]}\right) \cdot c\left(\gamma+\alpha_{n} \cdot \zeta_{n}^{-1}+\beta_{n} \cdot \zeta_{n}\right) \cdot P\left(j^{\star}\left(M^{[n]}\right)^{\vee}, B\right)$, where, as before,

$$
j:(\mathcal{C} / \mathcal{B})^{[n]} \rightarrow X^{[n]}
$$

Let $\mu$ denote one of the classes $\alpha_{1}, \ldots, \alpha_{\ell}$. Invoking (16), we have

$$
j^{\star} \mu^{[n]}=\bar{\mu}-\mu_{n} \cdot \zeta_{n}^{-1}
$$

and hence

$$
\operatorname{ch}_{k}\left(j^{\star} \mu^{[n]}\right)=\operatorname{ch}_{k}(\bar{\mu})-\sum_{i=0}^{k} \frac{(-1)^{k-i}}{(k-i) !} \cdot \operatorname{ch}_{i}\left(\mu_{n}\right) \cdot c_{1}\left(\zeta_{n}\right)^{k-i} .
$$

Following the derivation of equation (22), we obtain

$$
\int_{\mathbb{P}_{n}} c_{1}\left(\zeta_{n}\right)^{s} \cdot \rho_{n} \cdot \lambda \cdot c\left(\alpha_{n} \cdot \zeta_{n}^{-1}+\left(\beta_{n}\right)^{\vee} \cdot \zeta_{n}\right) \cdot P_{b_{1}}\left((-M)_{n}^{\vee} \cdot \zeta_{n}\right) \cdots P_{b_{m}}\left((-M)_{n}^{\vee} \cdot \zeta_{n}\right)
$$

Compared to (22), the extra terms are $c_{1}\left(\zeta_{n}\right)^{s}$ and the class $\rho_{n}$ which is a universal polynomial in the Chern classes

$$
c_{i}\left(\mu_{n}\right)
$$

where $\mu$ is one of the classes $\alpha_{1}, \ldots, \alpha_{\ell}$. These extra terms arise from the product expansion

$$
\operatorname{ch}_{k_{1}}\left(j^{\star} \alpha_{1}^{[n]}\right) \cdots \operatorname{ch}_{k_{\ell}}\left(j^{\star} \alpha_{\ell}^{[n]}\right)
$$

using (40). Crucially for us, $s$ and the $i$ 's are bounded from above by an expression that depends on $k_{1}, \ldots, k_{\ell}$. Thus they are independent of $n$.

The rest of the argument is as in Sections 1.3.3 and 1.3.7 we expand all expressions in powers of $c_{1}\left(\zeta_{n}\right)$ and integrate over the fibers of

$$
\mathbb{P}_{n} \rightarrow \mathfrak{M} .
$$


Keeping track of the numerical modifications is not difficult. The powers $c_{1}\left(\zeta_{n}\right)^{s}$ affect the indices of various sums defining the prefactors $\sigma^{(n)}$, see for instance (23). Since $s$ is fixed independently of $n$, the conclusions of Lemma 4 still hold. Furthermore, Lemma 5 can be applied to each of the additional terms $c_{j}\left(\mu_{n}\right)$ for $\mu$ being one of $\alpha_{1}, \ldots, \alpha_{\ell}$. In the end, (41) is still an expression of the form (因). Rationality is therefore established.

\section{REFERENCES}

[AK] A. Altman, S. Kleiman, Compactifying the Picard scheme, Advances in Math. 35 (1980), 50-112.

[AJLOP] N. Arbesfeld, D. Johnson, W. Lim, D. Oprea, R. Pandharipande, The virtual K-theory of Quot schemes of surfaces, J. Geom. Phys, DOI: 10.1016/j.geomphys.2021.104154.

[C] E. Carlsson, Vertex operators and quasimodularity of Chern numbers on the Hilbert scheme, Advances in Math. 229 (2012), 2888-2907.

[DKO] M. Duerr, A. Kabanov, Ch. Okonek, Poincare invariants, Topology 46 (2007), 225-294.

[EGL] G. Ellingsrud, L. Göttsche, M. Lehn, On the cobordism class of the Hilbert scheme of a surface, J. Alg. Geom. 10 (2001), 81-100.

[F] J. Fogarty, Algebraic Families on an Algebraic Surface, Am. J. Math. 10 (1968), 511-521.

[FG] B. Fantechi, L. Göttsche, Riemann-Roch theorems and elliptic genus for virtually smooth schemes, Geom. Topol. 14 (2010), 83-115.

[Fr] R. Friedman, Algebraic Sufaces and Holomorphic Vector Bundles, Springer-Verlag, New York (1998).

[G] I. Gessel, A combinatorial proof of the multivariable Lagrange inversion formula, J. Comb. Theory, Ser. A, 45 (1987), 178-195.

[Gö] L. Göttsche, The Betti numbers of the Hilbert scheme of points on a smooth projective surface, Math. Ann. 286 (1990), 193-207.

[GK1] L. Göttsche, M. Kool, Virtual refinements of the Vafa-Witten formula, Comm. Math. Phys. 376 (2020), 1-49.

[GK2] L. Göttsche, M. Kool, Refined SU(3) Vafa-Witten invariants and modularity, Pure and Appl. Math. Quart. 14 (2018), 467-513.

[GS] L. Göttsche, V. Shende, Refined curve counting on complex surfaces, Geom. Topol. 18 (2014), $2245-2307$.

[GP] T. Graber, R. Pandharipande, Localization of virtual classes, Invent. Math. 135 (1999), 487-518.

[Gr] I. Gronojnowski, Instantons and affine algebras I. The Hilbert scheme and vertex operators, Math. Res. Lett. 3 (1996), 275-291.

[KT] M. Kool, R. Thomas, Reduced classes and curve counting on surfaces I: Theory, Alg. Geom. 1 (2014), 334-383.

[Le] M. Lehn, Chern classes of tautological sheaves on Hilbert schemes of points on surfaces, Invent. Math. 136 (1999), 157-207.

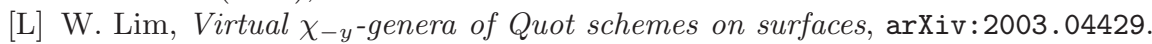

[MOP1] A. Marian, D. Oprea, R. Pandharipande, Segre classes and Hilbert schemes of points, Annales Scientifiques de l'ENS 50 (2017), 239-267.

[MOP2] A. Marian, D. Oprea, R. Pandharipande, The combinatorics of Lehn's conjecture, J. Math. Soc. Japan 71 (2019), 299-308.

[MOP3] A. Marian, D. Oprea, R. Pandharipande, Higher rank Segre integrals over the Hilbert scheme of points, J. Eur. Math. Soc (to appear), arXiv:1712.02382.

[MOOP] M. Moreira, A. Oblomkov, A. Okounkov, R. Pandharipande, Virasoro constraints for stable pairs on toric 3-folds, arXiv:2008.12514.

[N] H. Nakajima, Heisenberg algebra and Hilbert schemes of points on projective surfaces, Ann. Math. 145 (1997), 379-388.

[OOP] A. Oblomkov, A. Okounkov, R. Pandharipande, GW/PT descendent correspondence via vertex operators, Comm. Math. Phys. 374 (2020), 1321-1359.

[OP1] D. Oprea, R. Pandharipande, Quot schemes of curves and surfaces: virtual classes, integrals, Euler characteristics, Geom. Topol (to appear), arXiv:1903.08787. 
[OP2] D. Oprea, R. Pandharipande, private conversation, ETH Zurich, June 2019.

[P] R. Pandharipande, Descendents for stable pairs on 3-folds, Modern Geometry: A celebration of the work of Simon Donaldson, Proc. Sympos. Pure Math. 99 (2018), 251-288.

[PP1] R. Pandharipande, A. Pixton, Descendents for stable pairs on 3-folds: Rationality, Comp. Math. 149 (2013), 81-124.

[PP2] R. Pandharipande, A. Pixton, Descendent theory for stable pairs on toric 3-folds, J. Math. Soc. Japan 65 (2013), 1337-1372.

[PP3] R. Pandharipande, A. Pixton, Gromov-Witten/Pairs descendent correspondence for toric 3-folds, Geom. Topol. 18 (2014), 2747-2821.

[PT1] R. Pandharipande, R. Thomas, Curve counting via stable pairs in the derived category, Invent. Math. 178 (2009), 407-447.

[PT2] R. Pandharipande, R. Thomas, Stable pairs and BPS invariants, J. Amer. Math. Soc. 23 (2010), 267-297.

[R] S. di Rocco, k-very ample line bundles on del Pezzo surfaces, Math. Nach. 179 (1996), 47-56.

[T] R. P. Thomas, A holomorphic Casson invariant for Calabi-Yau 3-folds, and bundles on K3 fibrations, Jour. Diff. Geom. 54 (2000), 367-438.

[TT] Y. Tanaka, R. P. Thomas, Vafa-Witten invariants for projective surfaces I: stable case, J. Alg. Geom. 29 (2020), 603-668.

[V] C. Voisin, Segre classes of tautological bundles on Hilbert schemes of points of surfaces, Alg. Geom. 6 (2019), 186-195.

[VW] C. Vafa, E. Witten, A strong coupling test of S-duality, Nuclear Phys. B 431 (1994), 3-77.

Department of Mathematics, ETH Zürich

Email address: jared.johnson@math.ethz.ch

Email address: d.johnson@mathematics.byu.edu

Department of Mathematics, University of California, San Diego

Email address: doprea@math.ucsd.edu

Department of Mathematics, ETH ZÜRICH

Email address: rahul@math.ethz.ch 